\title{
FLUID SLOSHING IN RECTANGULAR VESSELS WITH SIDE-WALL BAFFLES USING CONFORMAL MAPPINGS OF MULTIPLY-CONNECTED DOMAINS
}

\author{
by M. R. Turner $\ddagger$ \\ (Department of Mathematics, University of Surrey, Guildford, Surrey GU2 7XH, UK) \\ [Received 06 November 2017. Revised 06 February 2018.]
}

\begin{abstract}
Summary
This paper focuses on the problem of inviscid, irrotational, incompressible fluid sloshing in a rectangular vessel with rigid, impermeable side-wall baffles, and investigates the feasibility of using time-dependent conformal mappings to numerically simulate the evolution of the unknown free-surface in fully-dynamic simulations. An algorithm which uses conformal mappings of a multiply-connected domain to relate the conjugate harmonic functions along the free-surface is documented, and kinematic results presented for a prescribed free-surface motion. The results show that the specific mapping for an infinite depth fluid has one free, within specific bounds, mapping parameter, while the mapping for finite depth fluids has two free mapping parameters. It is shown that having two free parameters gives a wider range of situations under which the conformal mapping can be computed, and it is concluded that the finite depth mapping should be used (in the appropriate limit) even for infinite depth simulations. Overall it is found that a computationally efficient algorithm can be devised to relate the conjugate harmonic functions along the free-surface of the flow domain.
\end{abstract}

\section{Introduction}

A simply connected domain with a suitably smooth boundary can be mapped conformally to the interior of the unit disc or to the periodic half-plane $\{\xi=\mu+\mathrm{i} \nu: \operatorname{Im}(\xi)<0\}$, as shown in Fig. 1(a). Mappings such as this, have applications in water wave problems, because one major advantage of this approach is that the real and imaginary parts of any complex analytical function evaluated on the boundary (know as conjugate harmonic functions), are related via the Hilbert transform. This makes the numerical evaluation of either conjugate harmonic function, given the other, particularly fast. In the case of a doubly-connected domain, see Fig. 1(b), the domain is mapped to the interior of an annulus or the infinite periodic strip. In this case the mapping is more complex, and it has a conformal invariant, known as the conformal modulus, which needs to be correctly identified such that the mapping is conformal $(1 ; 2)$. This conformal modulus is related to the ratio of the radii of the annulus. The conjugate harmonic functions on the inner and outer radii of the domain are linked together via the Hilbert-Garrick transformation $(3 ; 1 ; 2 ; 4)$ which again allows for fast, effective numerical computations of the conjugate harmonic functions.

The interest in the current paper is multiply-connect domains which can be mapped

$\ddagger$ Email: m.turner@surrey.ac.uk

Q. Jl Mech. Appl. Math. (2018) xx (y), 1-29

(C) Oxford University Press 2018 

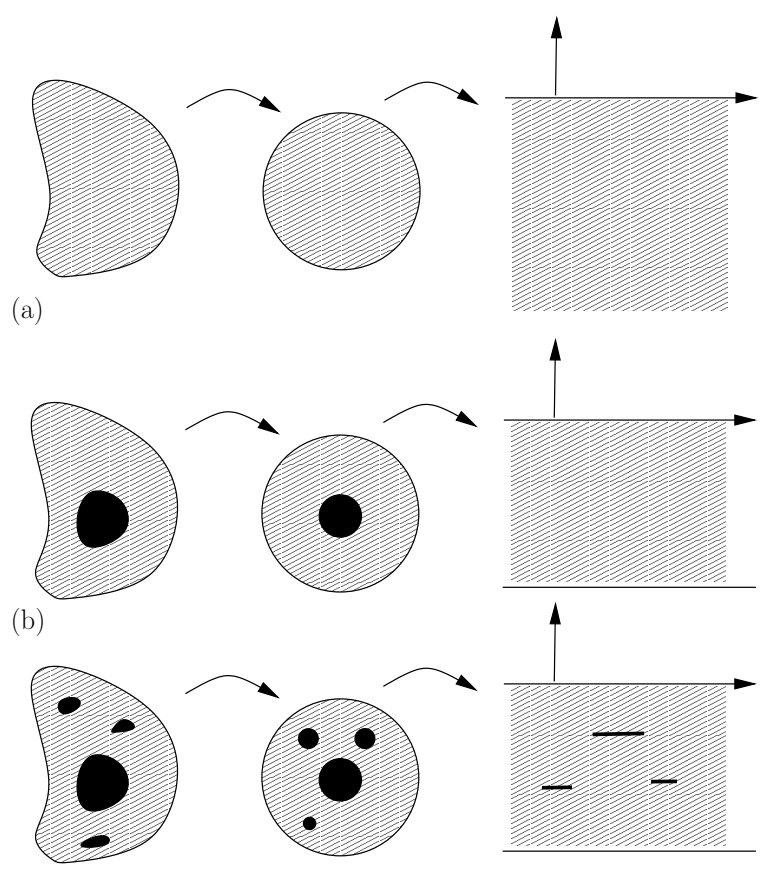

(c)

Fig. 1 (a) Conformal map of a simply-connected domain to the periodic half-plane, (b) conformal map of a doubly-connected domain to the periodic strip and (c) conformal map of a multiplyconnected domain to the periodic strip with horizontal baffles.

to the interior of the unit disc with multiple circular regions removed, or to the periodic half-plane with infinitely thin horizontal strips removed, see Fig. 1(c). In this paper we formulate a novel algorithm which allows one conjugate harmonic function on each of the disc boundaries to be determined given information about its associated function. The motivation for this is to ultimately compute the evolution of water waves with submerged horizontal baffles in a fast, effective manner. Note, in the multiply-connected case there are many conformal invariants to calculate, which are related to the centre positions and radii of the removed circular regions.

The particular water wave problem considered in this this paper is the 'sloshing' problem of a two-dimensional spatial wave in a bounded domain (5). The domain consists of a rectangular vessel with submerged horizontal baffles attached to the side walls, such as that depicted in the schematic diagram in Fig. 2. In such problems the position of the free-surface interface is not known a priori and evolves with time, thus making them computationally difficult (6). One approach used extensively in the corresponding problem without submerged baffles is to map the moving domain to a fixed domain, specifically a rectangular or circular domain, and of particular interest here are those mappings which are conformal. Time-dependent conformal mappings have been used extensively on water 


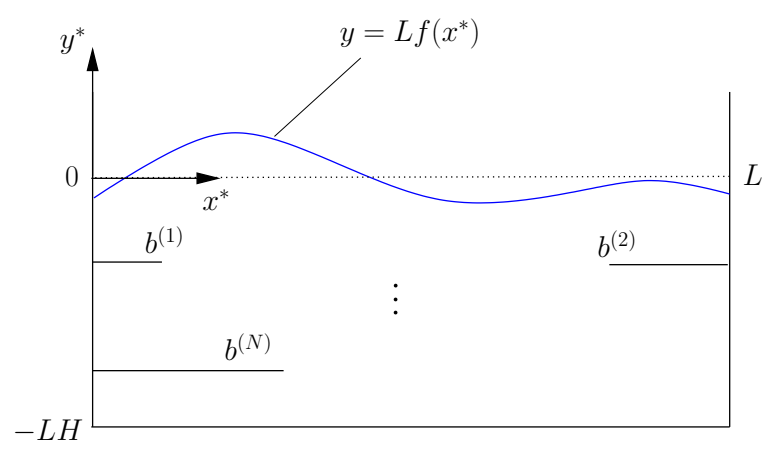

Fig. 2 Schematic dimensional diagram of $N$ horizontal submerged baffles connected to the sidewalls of a rectangular vessel. The dotted line signifies the undisturbed free-surface.

wave problems in both infinite depth $(7 ; 8 ; 9 ; 10 ; 11 ; 12 ; 13 ; 14 ; 15 ; 16 ; 17 ; 18)$ and finite depth $(14 ; 19 ; 20 ; 21 ; 22)$.

The baffled problem considered in Fig. 2 maps conformally to the multiply-connected circular domain in Fig. 1(c). As far as the author is aware there have been no studies investigating time-dependent mappings in this case. The main reason for this is because it has only been in the last decade or so that the theoretical and numerical mechanisms for constructing conformal maps to and from multiply-connected domains has been developed comprehensively via use of the Schottky-Klein prime function $(23 ; 24 ; 25 ; 26 ; 27)$. Of particular significance is the work of Crowdy et al. (28) who have developed a suite of numerical MATLAB ${ }^{\circledR}$ subroutines which readily evaluate these Schottky-Klein prime functions. Despite the existence of these routines the process for relating the conjugate harmonic functions on the domain boundary still remains complex and this process is the fundamental focus of this paper. While the motivation for this paper is to model twodimensional free-surface sloshing problems with submerged baffles, the method presented is more general and its application has a wider impact to problems which contain a periodically deformed surface with submerged horizontal boundaries.

In a time-dependent water wave problem, the mapping (i.e. the centre positions and radii of the removed discs in Fig. 1(c)) is time dependent, therefore as the position of the unknown free-surface is updated at each time-step, so the conjugate harmonic functions on the domain boundary need to be determined. In order to focus on the second part of this process in this paper, the calculation of the conjugate harmonic functions, we do not consider the full dynamic time-dependent problem, but instead we consider a parametric, kinematic problem where the free-surface is prescribed by a parameter, $t$, which mimics the time-dependence of the full problem. This will allow for a systematic study on the use of conformal mappings to solve the sloshing problem with side-wall baffles.

The paper is laid out as follows. In $\S 2$ the conformal mapping problem is set out, and the solution procedure in terms of Cauchy's integral theorem presented. The numerical procedure for solving the resulting equations is found in $\S 3$ and numerical results for both infinite depth and finite depth fluids are given in $\S 4$. Conclusions and a discussion of how this procedure helps us to solve the dynamic time-dependent problem are found in $\S 5$. 


\section{Mathematical Formulation}

We are interested in investigating the dimensional sloshing problem in a rectangular vessel depicted in the schematic diagram in Fig. 2. The problem consists of a free-surface elevation $y^{*}=L f\left(x^{*}\right)$, a solid bottom at $y^{*}=-L H$ and $N$ rigid, impermeable baffles, $b^{(n)} n=$ $1, \ldots, N$, attached to the vertical side walls of the vessel, each with an associated length $L L_{n}$ and at a depth $-L y_{n}$. Infinite depth fluid results, occur in the limit when $L H \rightarrow \infty$. Here the stars denote dimensional quantities. We choose to non-dimensionalize the system by setting $(x, y)=L^{-1}\left(x^{*}, y^{*}\right)$. In order to use the conformal mapping techniques presented in this paper we first extend the scenario in Fig. 2 to one which is periodic by forming the even extension of the physical problem from $x \in[0,1]$ to $x \in[-1,1]$ as depicted in Fig. 3(a). The unknown position of the free-surface $f(x)$, makes numerical computations difficult and hence we map the domain in the physical $z$-plane, where $z=x+\mathrm{i} y$, to a rectangular domain the $\xi$-plane, where $\xi=\mu+\mathrm{i} \nu$, given in Fig. $3(\mathrm{~b})$. In the $\xi$-plane $x=-1,0,1$ maps to $\mu=-1,0,1, y=-H$ maps to $\nu=-\widehat{H}$ and $y=f(x)$ maps to $\nu=0$. The upper and lower surfaces of each baffle $b^{(n)}$ get mapped to the upper and lower surfaces of the horizontal baffles $\widehat{b}^{(n)}$. Note that in this plane each baffle remains centred on either $\mu=0$ or $\mu=1$ to preserve the problem symmetry, but the baffles now have length $2 \widehat{L}_{n}$ and are positioned at $\nu=-\widehat{y}_{n}$. The case when $\widehat{L}_{n}=L_{n}$ and $\widehat{y}_{n}=y_{n}$ only occurs when $f(x)=0$ and thus the baffle lengths and depths in the $\xi$-plane need to be computed as part of the solution.

In the $\xi$-plane the physical coordinates are $x(\mu, \nu)$ and $y(\mu, \nu)$, satisfy the CauchyRiemann equations

$$
x_{\mu}=y_{\nu}, \quad \text { and } \quad x_{\nu}=-y_{\mu},
$$

in the $\xi$-plane and thus both satisfy Laplace's equation

$$
x_{\mu \mu}+x_{\nu \nu}=0, \quad y_{\mu \mu}+y_{\nu \nu}=0 \text {. }
$$

When these coordinates are evaluated on the free-surface $\nu=0$, they give a parametric representation of the free-surface

$$
\left(X_{0}(\mu), Y_{0}(\mu)\right)=(x(\mu, 0), y(\mu, 0)) \quad \text { for } \quad \mu \in[-1,1] .
$$

Similarly we obtain parametric representations for the baffles

$$
\left(X_{n}(\mu), Y_{n}(\mu)\right)=\left(x\left(\mu,-\widehat{y}_{n}\right), y\left(\mu,-\widehat{y}_{n}\right)\right), \quad \text { for } \quad n=1, \ldots, N,
$$

and $\mu \in\left[-\widehat{L}_{n}, \widehat{L}_{n}\right]$ for baffles centred at $\mu=0$ (left-hand wall baffles) and $\mu \in[-1,-1+$ $\left.\widehat{L}_{n}\right] \cup\left[1-\widehat{L}_{n}, 1\right]$ for baffles centred at $\mu=1$ (right-hand wall baffles). On $\nu=-\widehat{H}$ the bottom has the parametric form

$$
\left(X_{N+1}(\mu), Y_{N+1}(\mu)\right)=(x(\mu,-\widehat{H}), y(\mu,-\widehat{H})), \quad \text { for } \quad \mu \in[-1,1] .
$$

In order to use Cauchy's integral theorem to link the conjugate harmonic functions on the free-surface, bottom and baffles, we further map the $\xi$-plane conformally to the $\eta$-plane in Fig. 3(c) using

$$
\eta=\exp (-\mathrm{i} \pi \xi)
$$


(a)

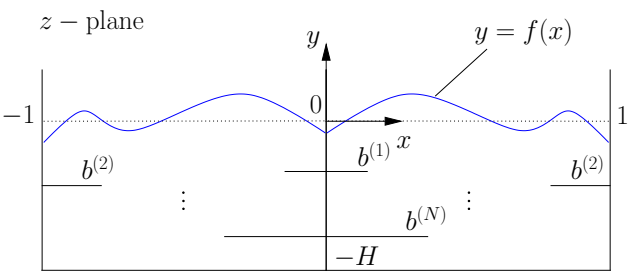

(c)

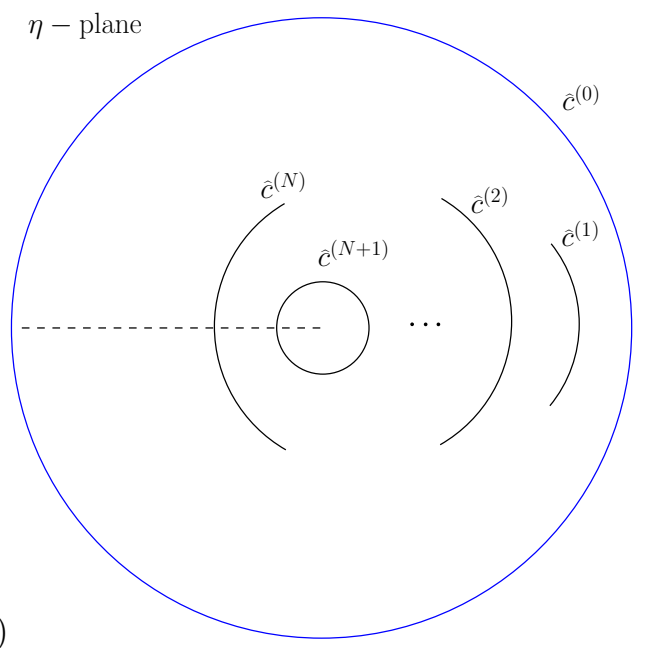

(b)

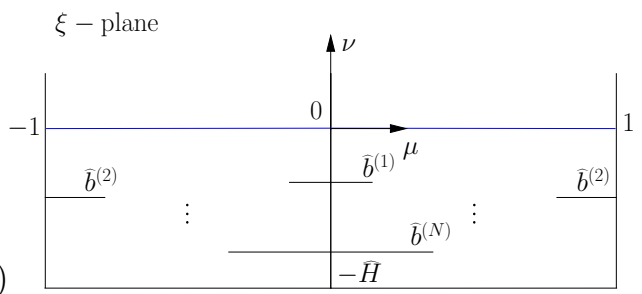

(d)

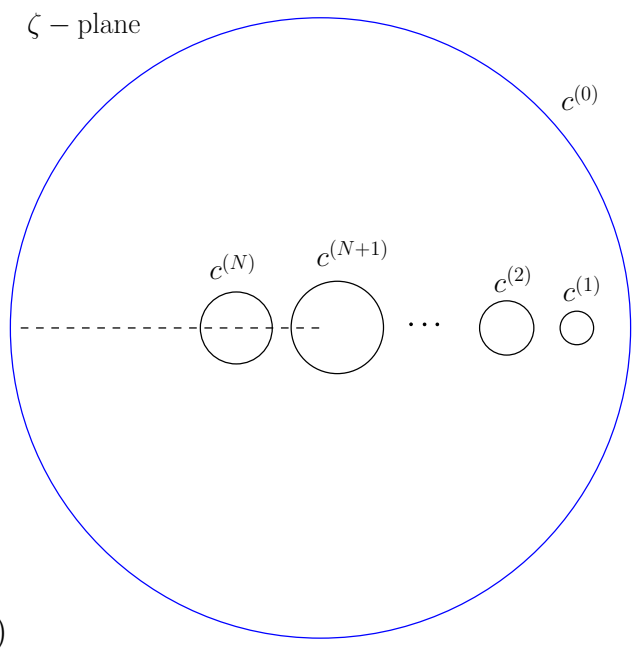

Fig. 3 Schematic diagrams depicting (a) the $z$-plane, (b) the $\xi$-plane, (c) the $\eta$-plane and (d) the $\zeta$-plane for the given problem in Fig. 2. The conformal mappings from (b) to (c) and (c) to (d) are given by (2.4) and (2.6) respectively. The dotted line in (a) denotes the undisturbed free-surface, while the dashed lines in (c) and (d) represent branch cuts separating different periodic windows of the flow.

In the $\eta$-plane the free-surface $\nu=0$ is mapped to the unit circle, $\widehat{c}^{(0)}$, the bottom $\nu=-\widehat{H}$ maps to the circle $\widehat{c}^{(N+1)}$, centered on the origin with radius $e^{-\pi \widehat{H}}$ and each baffle $b^{(n)}$ maps to an arc of a circle $\widehat{c}^{(n)}$, centered on the origin. In Fig. 3(c) the dashed line represents the branch cut linking different Riemann sheets, with $\mu=-1$ above the cut and $\mu=1$ below. Finally, the $\eta$-plane is conformally mapped to the $\zeta$-plane in Fig. 3(d) where Cauchy's integral theorem can be successfully applied. The exact form of this mapping depends upon whether we consider a finite depth or infinite depth fluid. In the case of an infinite depth fluid, the circle $\widehat{c}^{(N+1)}$ is not present, and this conformal mapping is the circular slit map

$$
\eta_{\text {inf }}(\zeta ; \alpha)=R_{0}(\zeta ; \alpha)=\frac{\omega(\zeta, \alpha)}{|\alpha| \omega\left(\zeta, \bar{\alpha}^{-1}\right)},
$$

where $\alpha$ is a constant mapping parameter (in fact it is the point in the $\zeta$-plane which maps to the origin in the $\eta$-plane) and $\omega(\cdot, \cdot)$ is the Schottky-Klein prime function for the particular multiply connected $\zeta$-plane $(29 ; 30 ; 23)$. In the finite depth case the conformal 
mapping from the $\zeta$-plane to the $\eta$-plane is given by

$$
\eta_{\text {fin }}(\zeta ; \alpha)=S_{0(N+1)}(\zeta ; \alpha)=\mathscr{B} \frac{R_{0}(\zeta ; \alpha)}{R_{N+1}(\zeta ; \alpha)},
$$

where

$$
R_{N+1}(\zeta ; \alpha)=\left[\frac{\omega(\zeta, \alpha) \bar{\omega}\left(\chi_{N+1}(\zeta), \chi_{N+1}(\alpha)\right)}{\omega\left(\zeta, \bar{\chi}_{N+1}(\bar{\alpha})\right) \bar{\omega}\left(\chi_{N+1}(\zeta), \bar{\alpha}\right)}\right]^{1 / 2}
$$

and

$$
\chi_{N+1}(\zeta)=\bar{\delta}_{N+1}+\frac{q_{N+1}^{2}}{\zeta-\delta_{N+1}}
$$

with $\delta_{N+1}$ and $q_{N+1}$ denoting the centre and radius of $c^{(N+1)}$ respectively, and the over-bars denoting the complex conjugate. The normalization constant $\mathscr{B}$ is defined such that the unit circle $c^{(0)}$ is mapped to the unit circle $\widehat{c}^{(0)}$. For full details on the above conformal mappings and for more details on the Schottky-Klein prime function the reader is referred to $(23 ; 27)$ and the references therein. Full details of these mappings are not required in order to construct the algorithm in this paper, we just need the fact that the mappings exist and can be numerically evaluated using MATLAB ${ }^{\circledR}$ subroutines, courtesy of (28).

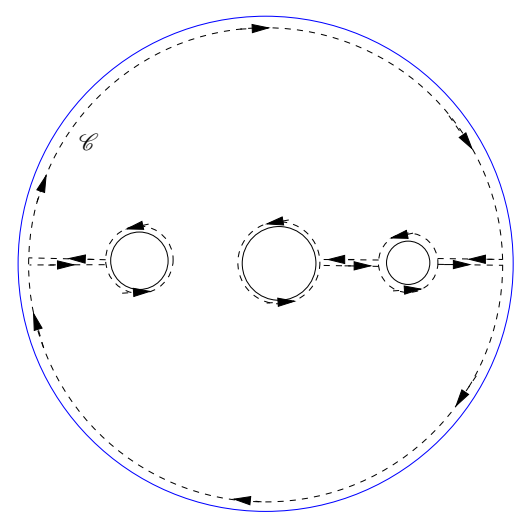

Fig. 4 The $\zeta$-plane with the dashed line depicting the closed contour $\mathscr{C}$ used to evaluate Cauchy's integral theorem.

In the $\zeta$-plane we apply Cauchy's integral theorem such that any complex analytical function $\kappa(\zeta)$ can be written as

$$
\kappa(\zeta)=-\frac{1}{2 \pi \mathrm{i}} \oint_{\mathscr{C}} \frac{\kappa\left(\zeta^{\prime}\right)}{\zeta^{\prime}-\zeta} \mathrm{d} \zeta^{\prime}
$$

where $\mathscr{C}$ is the clockwise traversed contour depicted in Fig. 4. For our problem the analytic function we consider is

$$
\kappa(\zeta)=(x(\zeta)-\mu(\zeta))+\mathrm{i}(y(\zeta)-\nu(\zeta))=\widetilde{x}(\zeta)+\mathrm{i} \widetilde{y}(\zeta) .
$$


Note, while $(\mu, \nu)=(\mu(\xi), \nu(\xi))$ implicitly, they can be considered as functions of either $\eta$ or $\zeta$ via the mappings (2.4) with either (2.5) or (2.6) respectively. For simplicity in notation we write $(\mu, \nu)=(\mu(\zeta), \nu(\zeta))$ explicitly with the understanding that $(2.5)$ and (2.6) have been applied to the values of $\xi$. The integrals along the parallel strips of the contour cancel out and (2.7) reduces to integrals over the individual circles $c^{(n)}$, each of which can be parameterized via

$$
\zeta^{\prime}=\delta_{n}+q_{n} e^{\mathrm{i} \theta}, \quad \theta \in[-\pi, \pi], \quad \text { for } \quad n=0 \text { and } N+1,
$$

and

$$
\zeta^{\prime}=\delta_{n}+q_{n} e^{\mathrm{i} \phi}, \quad \phi \in[0,2 \pi], \quad \text { for } \quad n=1, \ldots, N,
$$

where $\delta_{n}$ and $q_{n}$ are the centre and radius of $c^{(n)}$ respectively, with $\delta_{0}=0$ and $q_{0}=1$. Having exactly circular contours in the $\zeta$-plane has numerical advantages for evaluating the Cauchy-type integral in (2.7) and hence the use of the conformal mappings (2.5) and (2.6) is further justified. Thus in the $\zeta$-plane (2.7) becomes

$$
\begin{aligned}
\widetilde{x}(\zeta)+\mathrm{i} \widetilde{y}(\zeta)= & \frac{1}{2 \pi} \int_{-\pi}^{\pi} \frac{\widetilde{x}_{0}(\theta)+\mathrm{i} \widetilde{y}_{0}(\theta)}{e^{\mathrm{i} \theta}-\zeta} e^{\mathrm{i} \theta} \mathrm{d} \theta-\sum_{n=1}^{N} \frac{q_{n}}{2 \pi} \int_{0}^{2 \pi} \frac{\widetilde{x}_{n}(\phi)+\mathrm{i} \widetilde{y}_{n}(\phi)}{\delta_{n}+q_{n} e^{\mathrm{i} \phi}-\zeta} e^{\mathrm{i} \phi} \mathrm{d} \phi \\
& -\frac{q_{N+1}}{2 \pi} \int_{-\pi}^{\pi} \frac{\widetilde{x}_{N+1}(\theta)+\mathrm{i} \widetilde{y}_{N+1}(\theta)}{\delta_{N+1}+q_{N+1} e^{\mathrm{i} \theta}-\zeta} e^{\mathrm{i} \theta} \mathrm{d} \theta
\end{aligned}
$$

Therefore, in order to fully define the conformal mapping we need to firstly determine the centres and radii of the circles $c^{(n)} n=1, \ldots, N+1$ required to map the $\zeta$-plane to the configuration of baffles in the $\xi$-plane, and secondly to determine the functions $\widetilde{x}_{n}(s)$ and $\widetilde{y}_{n}(s)$ for $n=0, \ldots, N+1$ required to map to the original $z$-plane configuration. The process for determining the functions $\widetilde{x}_{n}$ and $\widetilde{y}_{n}$ involves evaluating (2.8) on the free-surface, each baffle and, in finite depth, the bottom and solving the resulting system of integral equations. For example, evaluating (2.8) on the free surface given in the $\zeta$-plane by $\zeta=e^{\mathrm{i} \sigma}$ for $\sigma \in[-\pi, \pi]$ leads to

$$
\begin{aligned}
& \widetilde{x}_{0}(\sigma)+\mathrm{i} \widetilde{y}_{0}(\sigma)=\frac{1}{2 \pi} \int_{-\pi}^{\pi}\left(\widetilde{x}_{0}(\theta)+\mathrm{i} \widetilde{y}_{0}(\theta)\right) \mathrm{d} \theta-\frac{\mathrm{i}}{2 \pi} \mathscr{P} \mathscr{V} \int_{-\pi}^{\pi} \cot \left[\frac{1}{2}(\theta-\sigma)\right]\left(\widetilde{x}_{0}(\theta)+\mathrm{i} \widetilde{y}_{0}(\theta)\right) \mathrm{d} \theta \\
& -\sum_{n=1}^{N} \frac{q_{n}}{\pi} \int_{-\pi}^{\pi} F_{n 0}(\sigma, \phi)\left(\widetilde{x}_{n}(\phi)+\mathrm{i} \widetilde{y}_{n}(\phi)\right) \mathrm{d} \phi-\frac{q_{N+1}}{\pi} \int_{-\pi}^{\pi} F_{(N+1) 0}(\sigma, \theta)\left(\widetilde{x}_{N+1}(\theta)+\mathrm{i} \widetilde{y}_{N+1}(\theta)\right) \mathrm{d} \theta,(2.9
\end{aligned}
$$

where

$$
F_{n m}(t, s)=\frac{e^{\mathrm{i} s}}{\delta_{n}+q_{n} e^{\mathrm{i} s}-\delta_{m}-q_{m} e^{\mathrm{i} t}},
$$

and the Sokhotski-Plemelj theorem is used to evaluate Cauchy's integral theorem on the boundary of the domain. The principal value integral in (2.9) is the representation of the Hilbert transform on the unit disc (2), and follows from (2.10) as

$$
F_{n n}(t, s)=\frac{1}{2 q_{n}}\left(1-\mathrm{i} \cot \left[\frac{1}{2}(s-t)\right]\right), \quad \forall n .
$$

Taking the real and imaginary parts of (2.9) gives two integral equations, which when 
combined with the equivalent integral equations from (2.8) evaluated on each baffle and the bottom, leads to $2 N+4$ equations which need to be satisfied by the functions $\widetilde{x}_{n}$ and $\widetilde{y}_{n}$ for $n=0, \ldots, N+1$. Writing out the full list of equations for the general $N$ baffle problem is cumbersome, but we display the six equations for the $N=1$ baffle case in a finite depth fluid in appendix A. In the equations presented in appendix A, we have halved each domain size by noting that we expect each $\widetilde{x}_{n}(s)$ to be an odd function about $\theta=0$ and $\phi=\pi$, and each $\widetilde{y}_{n}(s)$ to be an even function about this point. The reason for doing this is to halve the number of grid points required in the numerical scheme set out in $\S 3$, hence speeding up the numerical evaluation.

As the position of the baffles and impermeable bottom are fixed in the $z$-plane, and we assume a known free-surface elevation $y=f(x)$ (this assumption is consistent for the timedependent sloshing problems for which this conformal mapping approach is being devised), we need not solve for both the real and imaginary parts of $\kappa(\zeta)$ as $\widetilde{y}_{n}(s)$ for $n=0, \ldots, N+1$ will be known. Therefore we need only solve $N+2$ equations, allowing the remaining $N+2$ equations to be used to update the lengths and positions of the baffles, and the bottom position in the $\xi$-plane, $\widehat{y}_{n}, \widehat{L}_{n}$ and $\widehat{H}$. The solution for the conformal mapping has to be found numerically, and the algorithm for this is set out in $\S 3$.

\section{Numerical Approach}

The numerical process to calculate the conformal mapping for a given set of baffles $b^{(n)}$, a bottom depth $-H$ and a free-surface elevation $y=f(x)$, contains two main processes: determining the mapping from the $\eta$-plane to the $\zeta$-plane and then solving the integral equations of the form $(2.9)$ for $\widetilde{x}(s) n=0, \ldots, N+1$. The algorithm used in this paper is as follows:

\section{-START-}

1. Choose initial guesses for the values of $\widehat{y}_{n}, \widehat{L}_{n}$, for $n=1, \ldots, N$ and $\widehat{H}$ in the $\xi$-plane, and also for $n=0, \ldots, N+1$ the functions $\widetilde{x}_{n}$, and map this domain to the $\eta$-domain via (2.4).

2. Invert the conformal mapping (2.6) giving the values of $q_{n}, \delta_{n}$ for $n=1, \ldots, N+1$ and $\alpha$ in the $\zeta$-plane.

3. Calculate the values of $\widetilde{y}_{n}$ for $n=0, \ldots, N+1$ from the given information in the $z$-plane $\left(f(x), L_{n}, y_{n}\right.$ etc.), and solve the real parts of the integral equations $(2.9)$ for $\widetilde{x}_{n}$ for $n=0, \ldots, N+1$.

4. Use the imaginary parts of the integral equations from (2.9) to update the values of $\widehat{y}_{n}, \widehat{L}_{n}$ and $\widehat{H}$.

5. Repeat items 2, 3 and 4 until the algorithm converges.

$$
\text { - END- }
$$

We now look deeper into the the numerical processes behind steps 2 -4 above. To simplify the discussion we highlight these numerical processes for the case of one baffle $N=1$ in a finite depth fluid, for which the six integral equations are given in appendix A. 


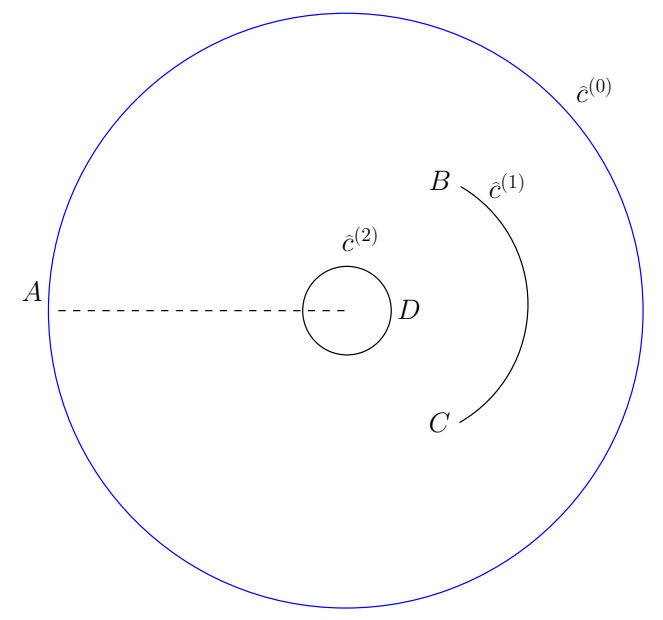

Fig. 5 The $\eta$-plane for the case $N=1$ baffle on the left-hand wall of the vessel in finite depth. The points $A, B, C$ and $D$ denote the parts of the $\xi$-plane which are used to determine the parameters in the $\zeta$-plane when numerically inverting (2.6). The dashed line represents the branch cut separating different periodic windows of the flow.

When we invert the conformal map (2.6) for $N=1$, we have 8 unknowns which need to be determined, namely $q_{1}, q_{2}, \delta_{1 r}, \delta_{1 i}, \delta_{2 r}, \delta_{2 i}, \alpha_{r}$ and $\alpha_{i}$ where the subscripts $r$ and $i$ denote the real and imaginary part respectively. These unknowns are found by using the 4 fixed values in the $\eta$-plane as shown in Fig. 5, namely the position of the branch cut $A$ (such that $\mu=-1$ above the cut and $\mu=1$ below), the real and imaginary parts of point $B$, the real or imaginary part of point $C$ and the radius of the circle corresponding to the bottom, $D$. As there are more unknowns than fixed points, we are free to choose (within certain limits) a set of these parameter values in the $\zeta$-plane. In this study we choose to set $\delta_{1 i}=\delta_{2 i}=\alpha_{i}=0$ as this fixes the point $A$, fixes point $C$ to be the complex conjugate of $B$, and gives a $\zeta$-plane circle configuration as in Fig. 3(d). Thus we have 5 remaining unknowns and 3 fixed values, therefore we are still free to choose two. It turns out that this is not a completely free choice and it is in fact restricted to a set of values from which a free choice can be made. In this study we choose to fix the baffle circle radius $q_{1}$ and mapping parameter $\alpha$ and determine the other 3 parameters. As the inverse of (2.6), $\eta_{\text {fin }}^{-1}$, has no analytic form, the 3 remaining parameters $q_{2}, \delta_{1 r}$, and $\delta_{2 r}$ are found via Newton iterations until updates to these values are less than $10^{-10}$ in magnitude. As we are choosing $\delta_{1}, \delta_{2}, \alpha \in \mathbb{R}$, we drop the subscript $r$ in the subsequent discussion with the implicit assumption they are real parameters.

In order to evaluate the functions $\widetilde{y}_{n}$ and solve the integral equations (A.2)-(A.7) for $\widetilde{x}_{n}$, $n=0,1,2$ we first discretize the domains $\sigma \in[-\pi, 0]$, both on the free-surface $\left(c^{(0)}\right)$ and bottom $\left(c^{(2)}\right)$, via

$$
\sigma_{j}=-\pi+\frac{\pi}{P}(j-1), \quad j=1, \ldots, P+1,
$$


and $\psi \in[0, \pi]$ on the baffle $\left(c^{(1)}\right)$ via

$$
\psi_{j}=\frac{\pi}{2 Q}(j-1), \quad j=1, \ldots, 2 Q+1 .
$$

Note, it is not necessary to have an equal number of grid points along both the free-surface and the bottom, but we choose to do so for simplicity. Similarly in the multiple baffle case, the discretization for each baffle could contain a different number of points.

The values of $\widetilde{y}_{n}\left(\sigma_{j}\right)=\widetilde{y}_{n j}$, for $n=0,2$ and $\widetilde{y}_{1}\left(\psi_{j}\right)=\widetilde{y}_{1 j}$ for each $j$ are then given by

$$
\begin{array}{lll}
\widetilde{y}_{0 j}=f\left(\mu_{j}+\widetilde{x}_{0 j}\right), & & j=1, \ldots, P+1, \\
\widetilde{y}_{1 j}=-y_{1}+\widehat{y}_{1}, & & j=1, \ldots, 2 Q+1, \\
\widetilde{y}_{2 j}=-H+\hat{H}, & j=1, \ldots, P+1,
\end{array}
$$

where $\mu_{j}=\mu\left(\sigma_{j}\right)$ evaluated on the free-surface. This furnishes $2 P+2 Q+3$ unknowns $\left(\widetilde{x}_{0 j}\right.$, $j=1, \ldots, P+1, \widetilde{x}_{1 j}, j=1, \ldots, 2 Q+1$ and $\left.\widetilde{x}_{2 j}, j=1, \ldots, P+1\right)$ to be calculated by solving the discretized form of the integral equations (A.2), (A.4) and (A.6). The discretized equations are evaluated at the mid-points of the domains

$$
\begin{array}{rlrl}
\sigma_{j}^{m} & =\frac{1}{2}\left(\sigma_{j}+\sigma_{j+1}\right), & j & =1, \ldots, P, \\
\psi_{j}^{m} & =\frac{1}{2}\left(\psi_{j}+\psi_{j+1}\right), & j=1, \ldots, 2 Q,
\end{array}
$$

as this allows the principle value integrals to be evaluated directly using the trapezoidal rule (31).

The discretized form of the equations (A.2)-(A.7) evaluated at (3.3) and (3.4) are given in (A.8)-(A.13) in appendix A. The $2 P+2 Q$ equations (A.8), (A.10) and (A.12) along with the conditions

$$
\widetilde{x}_{01}=\widetilde{x}_{11}=\widetilde{x}_{21}=0
$$

are solved via Newton iterations.

After each iteration, step 4 of the algorithm updates the values of $\widehat{y}_{1}, \widehat{L}_{1}$ and $\widehat{H}$ using the residues of (A.11) and (A.13) which we call $\widehat{R}_{2 j}$ and $\widehat{R}_{3 j}$ respectively, such that

$$
\begin{aligned}
\widehat{y}_{1}^{\dagger} & =\widehat{y}_{1}+\frac{1}{2} \operatorname{sgn}\left(\widehat{R}_{2 j}\right) \max \left(\left|\widehat{R}_{2 j}\right|\right), \\
\widehat{H}_{1}^{\dagger} & =\widehat{H}_{1}+\frac{1}{2} \operatorname{sgn}\left(\widehat{R}_{3 j}\right) \max \left(\left|\widehat{R}_{3 j}\right|\right), \\
\widehat{L}_{1}^{\dagger} & =\left\{\begin{array}{l}
\widehat{L}_{1}+\frac{1}{2}\left(\mu_{J}+\widehat{x}_{1 J}+L_{1}\right), \quad \text { left-wall baffle } \\
\widehat{L}_{1}+\frac{1}{2}\left(\mu_{J}+\widehat{x}_{1 J}+L_{1}-1\right), \quad \text { right-wall baffle }
\end{array}\right.
\end{aligned}
$$

where $\mu_{J}=\mu\left(\psi_{J}\right)$ is the point on the baffle where $\mu\left(\psi_{j}\right)$ obtains its minimum value. The $\dagger$ on the variables in (3.5)-(3.7) denotes the updated value at the next iteration. The Newton iteration for the solution for $\widetilde{x}_{n j}$ and updates for $\widehat{y}_{1}, \widehat{H}$ and $\widehat{L}_{1}$ continue until the maximum tolerance between updates of these quantities is less than $10^{-6}$. 
Note in (3.7) we find it more appropriate to update $\widehat{L}_{1}$ via this approach rather than using the residues $\widehat{R}_{1 j}$, but this alternative approach could be used. In the current algorithm setup, the maximum value of $\widehat{R}_{1 j}$ is a measure for the accuracy of the solution, hence we want to choose enough grid points $P, Q$ such that $\max \left(\left|\widehat{R}_{1 j}\right|\right)=O\left(10^{-6}\right)$ which is the same error magnitude we allow for the other residues.

Numerical results for various physical scenarios are given in $\S 4$.

\section{Results}

To demonstrate the conformal mapping approach outlined in $\S 2$ and $\S 3$ and to highlight the dependence of the mapping parameters on the form of the free-surface $f(x)$, we present numerical results in this section for the free-surface profile

$$
f(x)=f(x ; t)=\epsilon \cos (\pi x) \cos (t), \quad x \in[-1,1],
$$

where $\epsilon$ is an amplitude parameter. This profile is the lowest order free-sloshing mode in the unbaffled problem, and the parameter $t$ is synonymous with time in the dynamic problem. In the results which follow, we will consider $t=0$, except when demonstrating the dependence of the mapping parameters on the free-surface shape.

\subsection{Infinite Depth}

For the scenario of $N=1$ baffle in an infinite depth fluid, the $\zeta$-plane consists solely of the unit circle with an interior circle, $c^{(1)}$, removed. The convergence qualities of the numerical algorithm from $\S 3$ are shown in Fig. 6 for the case $L_{1}=1 / 3, y_{1}=-1 / 3$ and $q_{1}=0.166$ for a baffle on the left-hand wall. Here we plot the $\max \left(\left|\widehat{R}_{1 j}\right|\right)$ with $Q=P$ for $\epsilon=10^{-4}$ (result 1) and $\epsilon=10^{-2}$ (result 2). This shows that the scheme converges $\propto P^{-2}$ for both free-surface amplitudes considered. In dynamic simulations, such as in $(34 ; 4 ; 33)$, the largest amplitudes achievable in those simulations before overturning occurs is $O\left(10^{-2}\right)$ so this is the largest amplitude for which would wish this conformal mapping approach to work, hence is the largest amplitude we consider here. For larger amplitude waves with steep interfaces, the conformal mapping approach becomes less efficient as equally spaced grid points in the $\zeta$-plane become concentrated around the crests. In these type of problems a more efficient method would be the boundary element method (32). The residue $\widehat{R}_{2 j}$ is used to update $\widehat{y}_{1}$, and as we assume convergence when the iterative updates are $<10^{-6}$, we really only need to consider a value of $P$ such that $\max \left(\left|\widehat{R}_{1 j}\right|\right)<10^{-6}$. Hence for all results presented here we consider $Q=P=500$ and the results presented have converged to graphical accuracy. An example of the $\zeta_{-}, \eta$ - and $z$-plane for the case 1 in table 1 along with a depiction of the conformal mapping is given in Fig. 7.

The mapping plotted in Fig. 7 is for the case when $q_{1}=0.166$, but as mentioned in $\S 2$, there is a range of $q_{1}$ values for which the mapping exists, this range is plotted in Fig. 8 for $\epsilon=10^{-4}$ and $t=0$. The results in Fig. 8 show that the range of possible $q_{1}$ values is restricted due to $\alpha$ becoming zero at $q_{1} \approx 0.1658$ and because $q_{1}=\delta_{1}$ at $q_{1} \approx 0.1817$. This shows that in the mapping, $\alpha$ cannot move through the origin, nor can the circle $c^{(1)}$ encompass the origin. We will see later that this range is actually further restricted for the time-dependent problem. 


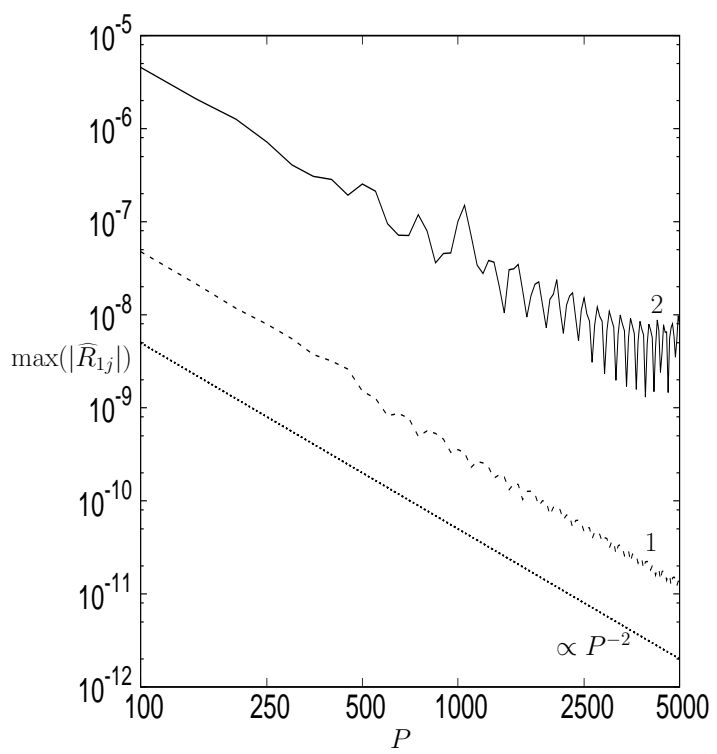

Fig. 6 Error residue $\max \left(\left|\widehat{R}_{1 j}\right|\right)$ as a function of $P=Q$ for the scenario $N=1$ baffle in infinite depth for $\epsilon=10^{-4}$ (result 1 ) and $\epsilon=10^{-2}$ (result 2). Here $L_{1}=1 / 3, y_{1}=-1 / 3, q_{1}=0.166$ and $t=0$ in (4.1).

\begin{tabular}{|c|r|r|r|r|r|}
\hline Parameter & Case 1 & Case 1.1 & Case 2 & Case 3 & Case 4 \\
\hline$L_{1}$ & 0.3333 & 0.3333 & 0.3333 & 0.3333 & 0.5000 \\
$y_{1}$ & -0.3333 & -0.3333 & -0.2500 & -0.5000 & -0.3333 \\
$q_{1}$ & 0.1660 & 0.1740 & 0.2200 & 0.1030 & 0.2290 \\
$\widehat{L}_{1}$ & 0.3303 & 0.3303 & 0.3294 & 0.3315 & 0.4964 \\
$\widehat{y}_{1}$ & -0.3358 & -0.3358 & -0.2533 & -0.5014 & -0.3350 \\
$\delta_{1}$ & 0.3165 & 0.2413 & 0.3433 & 0.1535 & 0.3021 \\
$\alpha$ & -0.0244 & -0.1083 & -0.1051 & -0.0535 & -0.0320 \\
\hline
\end{tabular}

Table 1 Table of parameters for $N=1$ left-hand wall baffle in infinite depth with $\epsilon=10^{-2}$ and $t=0$ in (4.1).

Fig. 9 plots the variation of the mapping parameters $\delta_{1}$ and $\alpha$ for the cases $1-4$ in table 1 as a function of the free-surface amplitude, $\epsilon$. In each case both $\delta_{1}$ and $\alpha$ reduce linearly with increasing $\epsilon$ at very similar rates, except for the longer baffle case, case 4 . This variation of $\delta_{1}$ and $\alpha$ is expected, because as $\epsilon$ increases the free-surface in the $z$-plane moves further from the baffle (when $t=0$ and the baffle is situated on the left-hand wall, $x=0$ ), hence the space between the baffle $c^{(1)}$ and the free-surface $c^{(0)}$ in the $\zeta$-plane should also increase. If we were to consider $t=\pi$ in (4.1), then as $\epsilon$ increases the spacing between the baffle and the free-surface would reduce for baffles on the left-hand wall and hence $\delta_{1}$ and $\alpha$ would be increasing functions of $\epsilon$. This case would mean $\alpha$ approaching zero, which we know is 
(a)
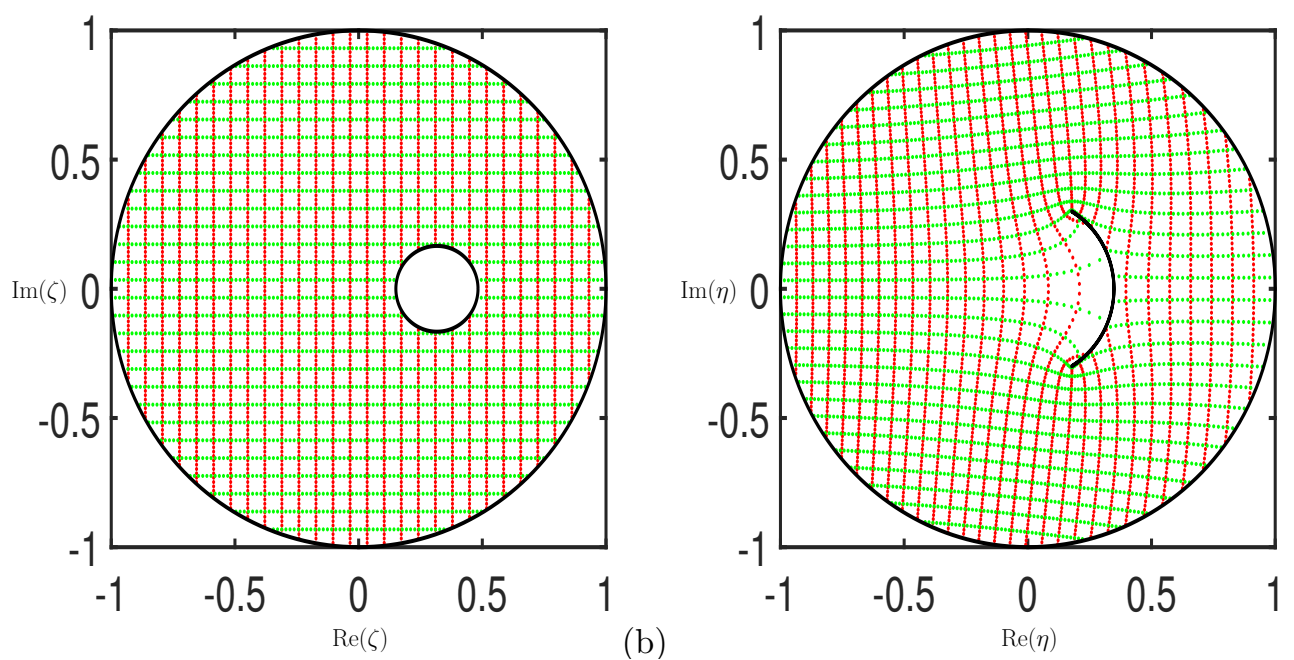

(b)

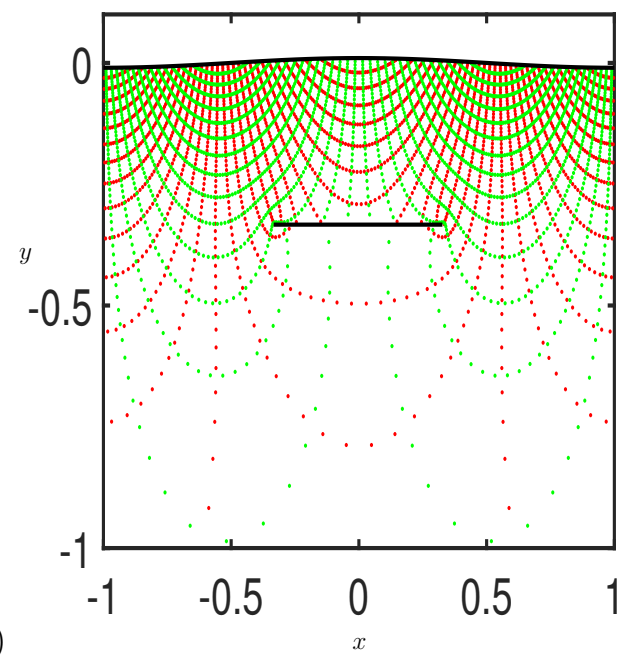

Fig. 7 Plot of (a) the $\zeta$-plane, (b) the $\eta$-plane and (c) the $z$-plane showing the conformal mapping for the scenario $N=1$ baffle in infinite depth for $\epsilon=10^{-2}$ and $t=0$ in (4.1). The parameters for this scenario are given by case 1 in table 1 .

problematic from Fig. 8(a). The resulting solutions for $\widetilde{x}_{0}(\theta)$ and $\widetilde{x}_{1}(\phi)$ are given in Fig. 10. At first glance they appear almost sinusoidal, but higher order terms are also present, in particular case 4 in Fig. 10(a) has a steeper gradient around $\theta=0$. The results for $\widetilde{x}_{1}(\phi)$ implicitly give the value of $\widehat{L}_{1}-L_{1}$ for each case, as the results are constrained such that $\max \left(\widehat{L}_{1}+\widetilde{x}_{1}(\phi)\right)=L_{1}$, hence $\max \left(\widetilde{x}_{1}(\phi)\right)=L_{1}-\widehat{L}_{1}$. Thus the longer baffle in case 4 shortens more in the $\xi$-plane than the result in case 1 which occurs at the same depth 
(a)

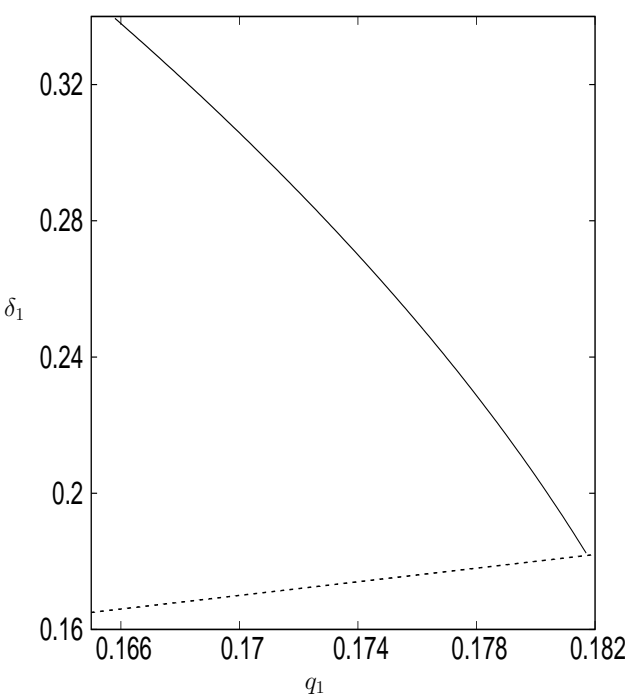

(b)

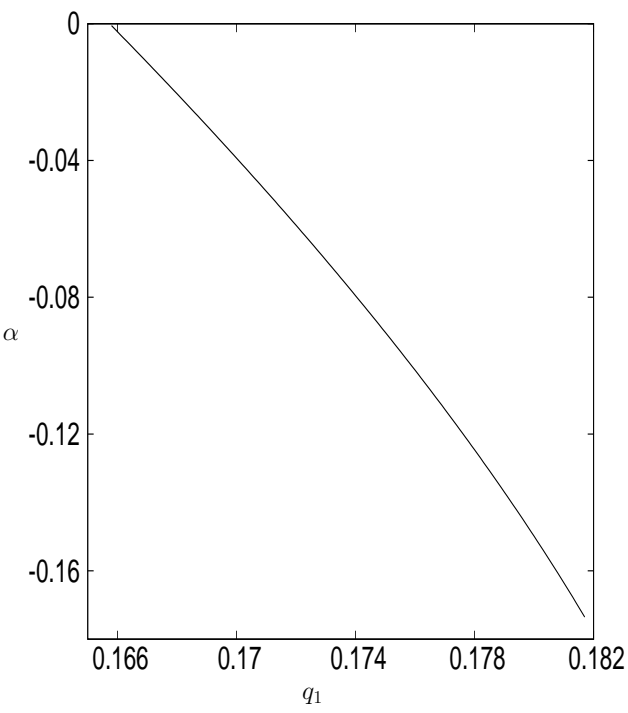

Fig. 8 Plot of (a) $\delta_{1}\left(q_{1}\right)$ and (b) $\alpha\left(q_{1}\right)$ for the conformal mapping for the scenario $N=1$ baffle in infinite depth, with $\epsilon=10^{-4}$ and $t=0$ in (4.1). The dashed line in panel (a) denotes the line $\delta_{1}=q_{1}$.
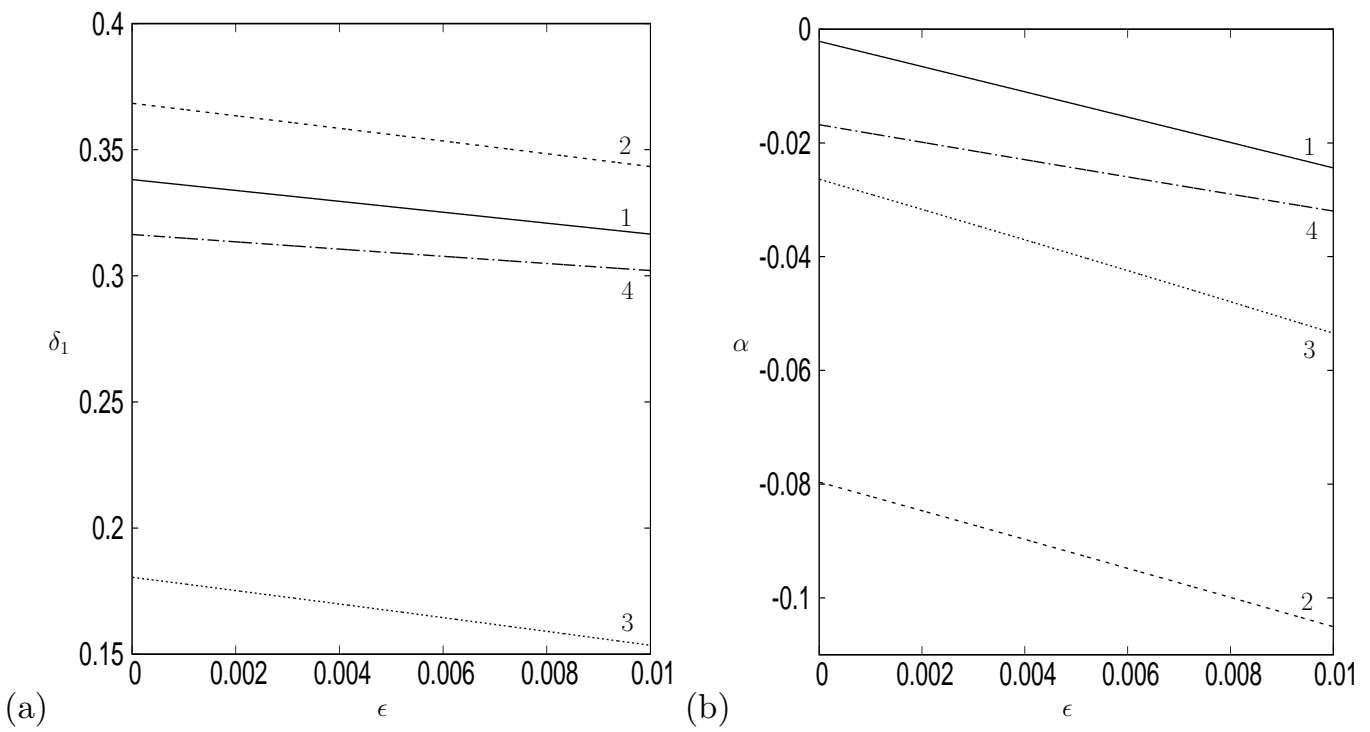

Fig. 9 Plot of (a) $\delta_{1}(\epsilon)$ and (b) $\alpha(\epsilon)$ for the conformal mapping for the scenario $N=1$ baffle in infinite depth with $t=0$ in (4.1). In each panel the results numbered 1-4 correspond to the cases 1-4 in table 1 . 

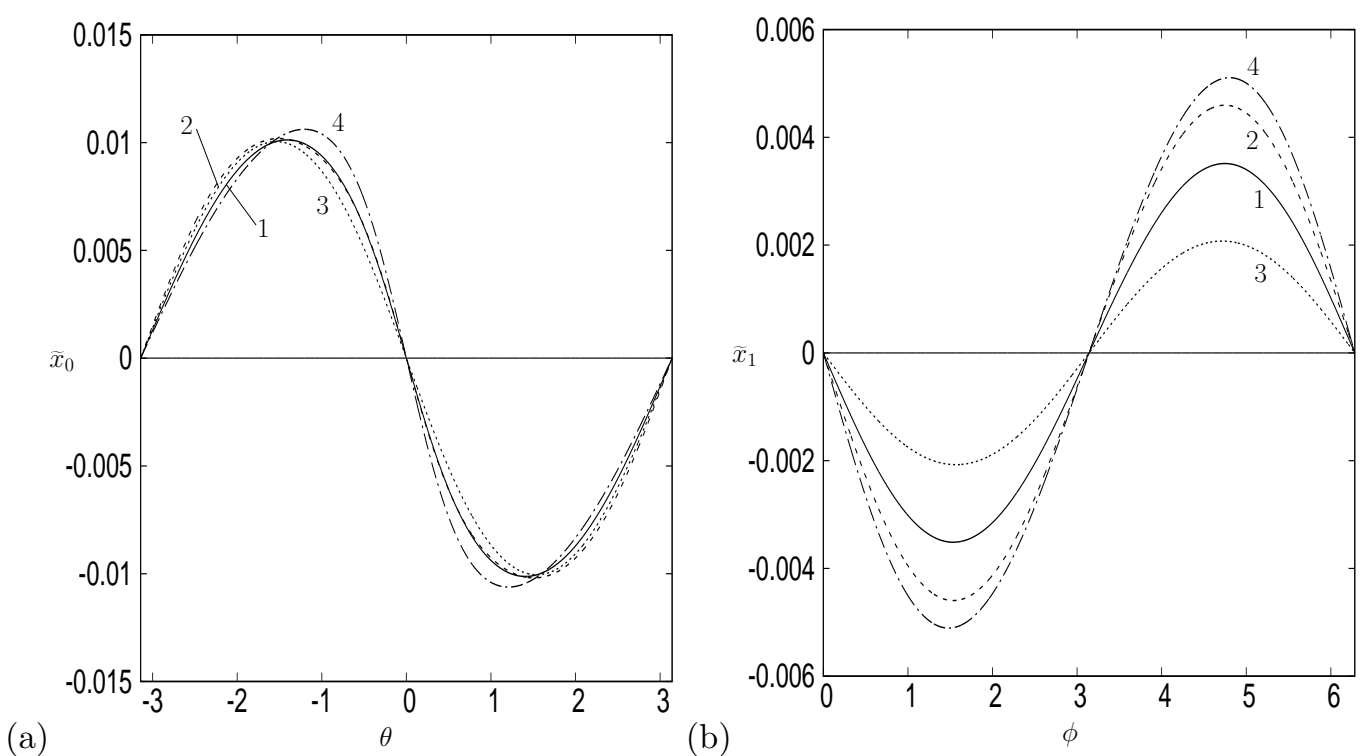

Fig. 10 Plot of (a) $\widetilde{x}_{0}(\theta)$ and (b) $\widetilde{x}_{1}(\phi)$ for the conformal mapping for the scenario $N=1$ baffle in infinite depth, with $\epsilon=10^{-2}$ and $t=0$ in (4.1). In each panel the results numbered 1-4 correspond to the cases $1-4$ in table 1 .

$y_{1}=-1 / 3$. However the percentage change between the two cases is very similar, with the $\max \left(\widetilde{x}_{1}(\phi)\right) \approx \epsilon L_{1}$ for these two cases.

The main reason for carrying out this feasibility study into the conformal mappings presented in this paper is to determine whether or not they could be applied to the timedependent dynamic sloshing problem with side-wall baffles. In order to understand whether or not this is possible, we consider the time evolution of (4.1) from $t=0$ to $t=\pi$, this is equivalent to simulating a wave sloshing over half a period. This would capture the two extremes of the free-surface position at $t=0$ and $t=\pi$ and checks that mappings can be found for all parameter values in between. The results for $\delta_{1}(t), \alpha(t), \widehat{L}_{1}(t)$ and $\widehat{y}_{1}(t)$ are given in Fig. 11 for the cases in table 1. The results show that all 4 parameters are clearly time-dependent and move to more positive values as the wave sloshes away from the left-hand side-wall of the vessel containing the baffle. Although the results appear to have rotational symmetry about $t=\pi / 2$ this is not the case and the parameters move a different magnitude depending whether the free-surface is above or below the mean quiescent level above the baffle.

The interesting result here is the case 1 result, which ceases just after $t=\pi / 2$, at $t=1.634$, at which point $\alpha \approx 0$. Therefore this value of $q_{1}$ would not be suitable to simulate the time-dependent sloshing problem with $\epsilon=10^{-2}$. However, when we increase this value to $q_{1}=0.174$ (case 1.1) we are able to solve for the conformal mapping parameters up to $t=\pi$. Therefore it appears that the conformal mapping approach presented here is feasible to use in the dynamic problem, but it is sensible to check the kinematic problem presented here first to find a suitable value of the parameter that is to be fixed. Another 

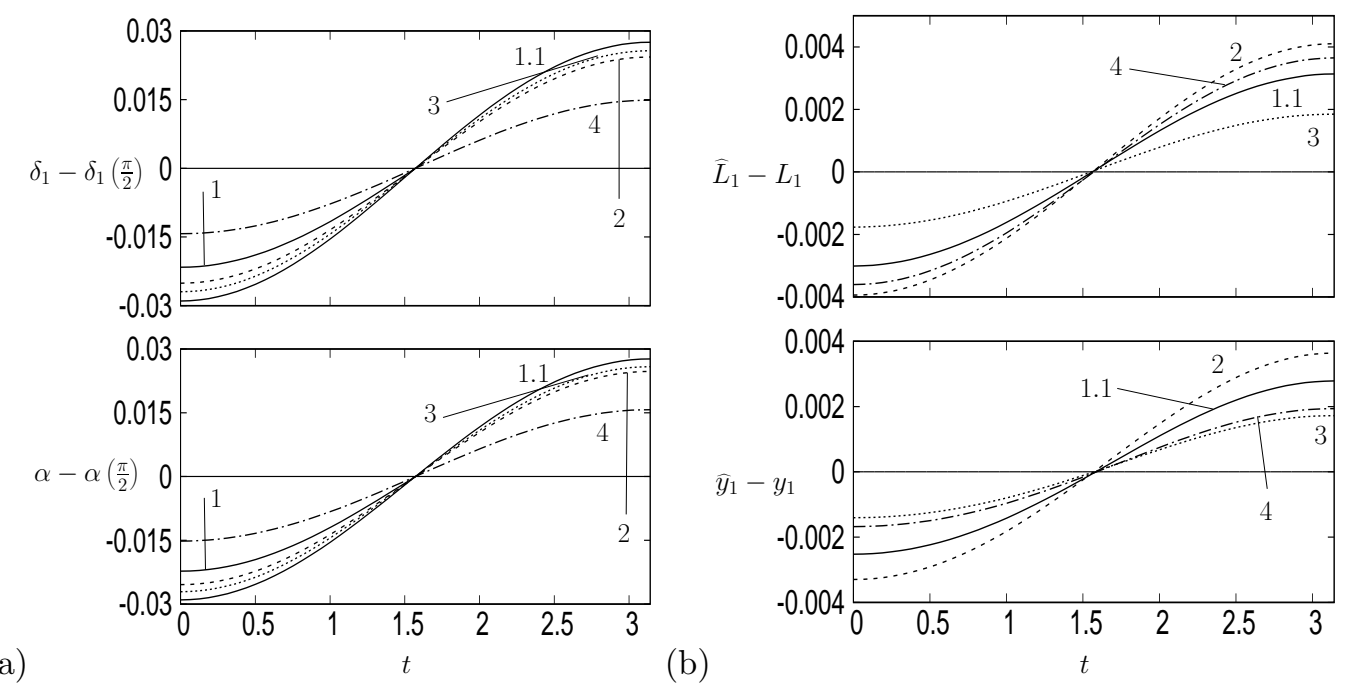

Fig. 11 Plot of (a) $\delta_{1}(t)-\delta_{1}(\pi / 2)$ and $\alpha(t)-\alpha(\pi / 2)$ and (b) $\widehat{L}_{1}(t)-L_{1}$ and $\widehat{y}_{1}(t)-y_{1}$ for the conformal mapping for the scenario $N=1$ baffle in infinite depth, with $\epsilon=10^{-2}$ for the cases given in table 1 .

\begin{tabular}{|c|r|r|r|r|}
\hline Parameter & Case 5 & Case 6 & Case 7 & Case 8 \\
\hline$L_{1}$ & $0.3333(l)$ & $0.2500(l)$ & $0.3333(l)$ & $0.3333(l)$ \\
$y_{1}$ & -0.3333 & -0.5000 & -0.3333 & -0.3333 \\
$L_{2}$ & $0.1667(l)$ & $0.3333(l)$ & $0.1667(r)$ & $0.5000(r)$ \\
$y_{2}$ & -0.2500 & -0.2500 & -0.4000 & -0.4000 \\
$q_{1}$ & 0.1550 & 0.0458 & 0.1700 & 0.1700 \\
$q_{2}$ & 0.1030 & 0.2274 & 0.0682 & 0.1577 \\
$\widehat{L}_{1}$ & 0.3303 & 0.2487 & 0.3303 & 0.3302 \\
$\widehat{y}_{1}$ & -0.3358 & -0.5015 & -0.3358 & -0.3356 \\
$\widehat{L}_{2}$ & 0.1640 & 0.2963 & 0.1681 & 0.5029 \\
$\widehat{y}_{2}$ & -0.2537 & -0.2535 & -0.3972 & -0.3985 \\
$\delta_{1}$ & 0.1048 & -0.3027 & 0.2658 & 0.0660 \\
$\delta_{2}$ & 0.3949 & 0.0480 & -0.3160 & -0.4158 \\
$\alpha$ & -0.2146 & -0.4335 & -0.0715 & -0.2045 \\
\hline
\end{tabular}

Table 2 Table of parameter quantities for $N=2$ baffles in infinite depth with $\epsilon=10^{-2}$ and $t=0$ from (4.1). The $(l)$ and $(r)$ in rows 1 and 3 signify whether the baffle is located on the left-hand wall or right-hand wall respectively.

approach may be to make $q_{1}$ time-dependent in such a way that $\alpha$ is moved away from zero if it becomes too close.

In Fig. 12 (and table 2) we consider cases 5-8 which contain two baffles either on the same wall or on different walls of the vessel. All the mappings have a very similar structure and 
(a)
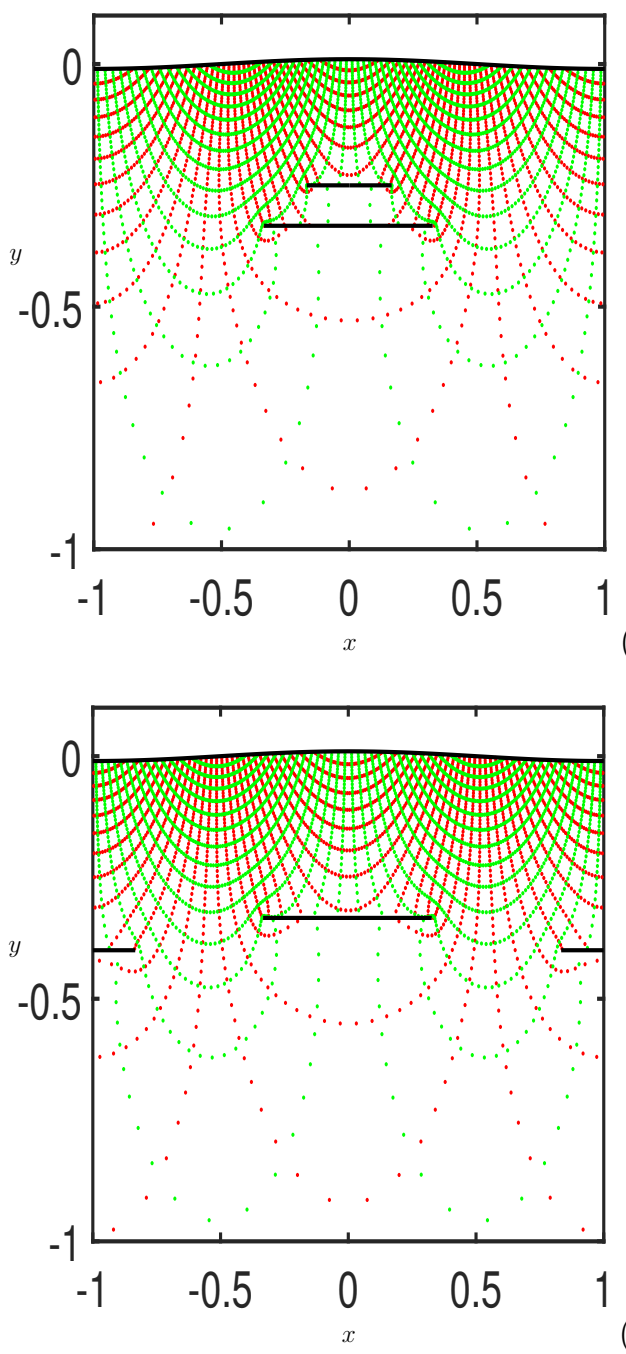

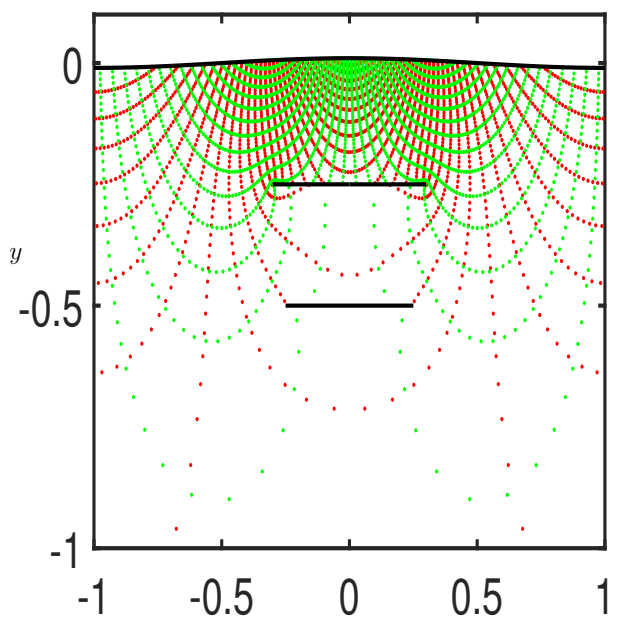

(b)

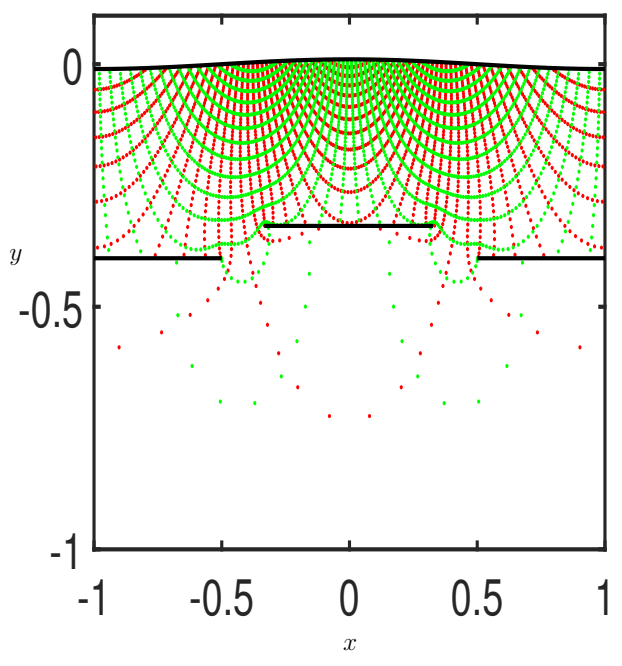

Fig. 12 Plot of the $z$-plane, showing the conformal mapping for the scenario $N=2$ baffles in infinite depth for $\epsilon=10^{-2}$ and $t=0$ in (4.1). The panels (a)-(d) correspond to the cases $5-8$ in table 2 respectively.

the interaction between the baffles is more apparent as the baffles become closer together as can be seen in the gaps between the baffles in Fig. 12(a) and 12(d). In each of the cases in Fig. 12 the dependence of the parameters on $\epsilon$, for example, are similar to those as in the 1 baffle case so we do not present those results here for two baffles, but what we are interested in, is whether or not we are able to simulate time-dependent dynamic sloshing over the half-period of the flow. The results for the time-dependence of the $\zeta$-plane parameters $\delta_{1}, \delta_{2}, q_{2}$ and $\alpha$ for cases 5-8 are plotted in Fig. 13. The results are very similar to those for $N=1$ baffle, but with some interesting differences. The results again do not 

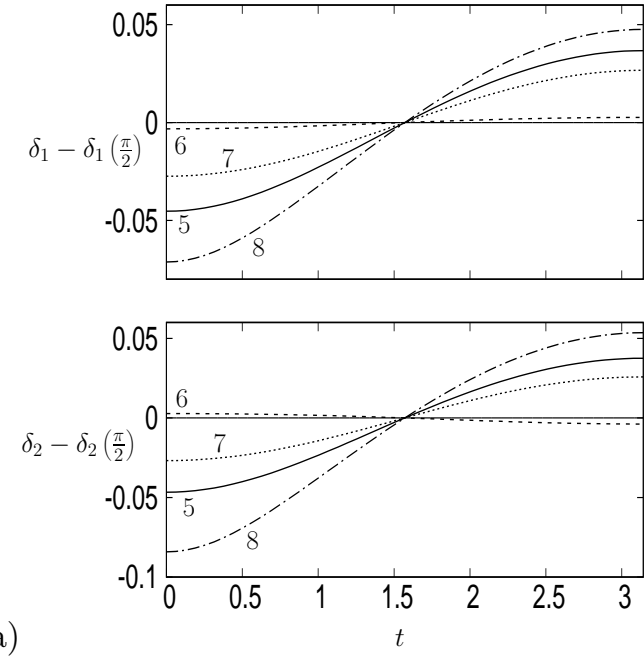
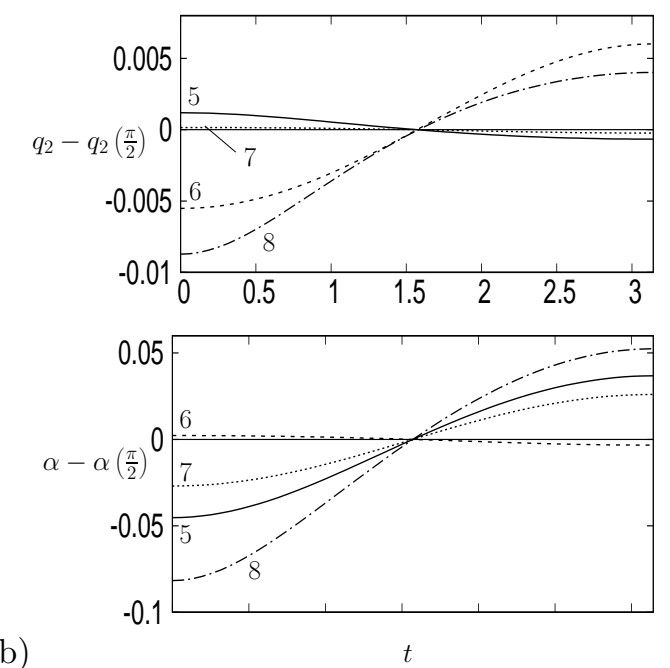

Fig. 13 Plot of (a) $\delta_{1}(t)-\delta_{1}(\pi / 2)$ and $\delta_{2}(t)-\delta_{2}(\pi / 2)$ and (b) $q_{2}(t)-q_{2}(\pi / 2)$ and $\alpha(t)-\alpha(\pi / 2)$ for the conformal mapping for the scenario $N=2$ baffles in infinite depth, with $\epsilon=10^{-2}$ for the cases given in table 2 .

have rotational symmetry about $t=\pi$ and in cases 5,7 and $8, \delta_{1}, \delta_{2}$ and $\alpha$ all increase as $t$ increases. However, one interesting case is case 6 , where $\delta_{1}$ increases while $\delta_{2}$ and $\alpha$ decrease for increasing $t$, but the magnitude of this movement is small. The top panel of Fig. 13(b) shows that it is the large variation of $q_{2}$, relative to the other parameters, which modifies the mapping in this case. Case 8 has parameters which vary the most in the $\zeta$-plane over one period of the flow, and this is due to the larger interaction between the two baffles compared to the other cases. The significant conclusion to the results presented in Fig. 13 is that for the fixed values of $q_{1}$ used, we are able to find conformal maps for $t \in[0, \pi]$, i.e. there are no cases where the numerical scheme breaks down. This shows that this method can be used for the dynamic problem in the general case in infinite depth fluids. We now consider finite depth fluids in $§ 4.2$ to understand the effect, if any, this has on the mapping results given in this section.

\subsection{Finite Depth}

When we consider finite depth fluids, the only change to the theoretical analysis is the conformal mapping from the $\zeta$-plane to the $\eta$-plane is now given by (2.6) instead of (2.5). The first question to consider is how do the parameters of this mapping compare with those of the infinite depth mapping (2.5) as $H \rightarrow \infty$ ? The answer to this question is given in Fig. 14 , where we again consider the $N=1$ baffle scenario given by case 1 in table 1 . What we find is that as $H \rightarrow \infty, \delta_{1} \rightarrow 0.3165$ (given by the upper dotted line), which is the same value as in the infinite depth calculation, and $q_{2} \rightarrow 0$, which we should expect, as we wish the radius of $\widehat{c}^{(2)}$ to tend to zero to be consistent with (2.4). However, what is interesting is that $\delta_{2} \rightarrow-0.0244$, which is the value of $\alpha$ from the infinite depth calculation (lower dotted line), thus $c^{(2)}$ in the finite depth mapping (2.6) plays the same role in the mapping as $\alpha$ 

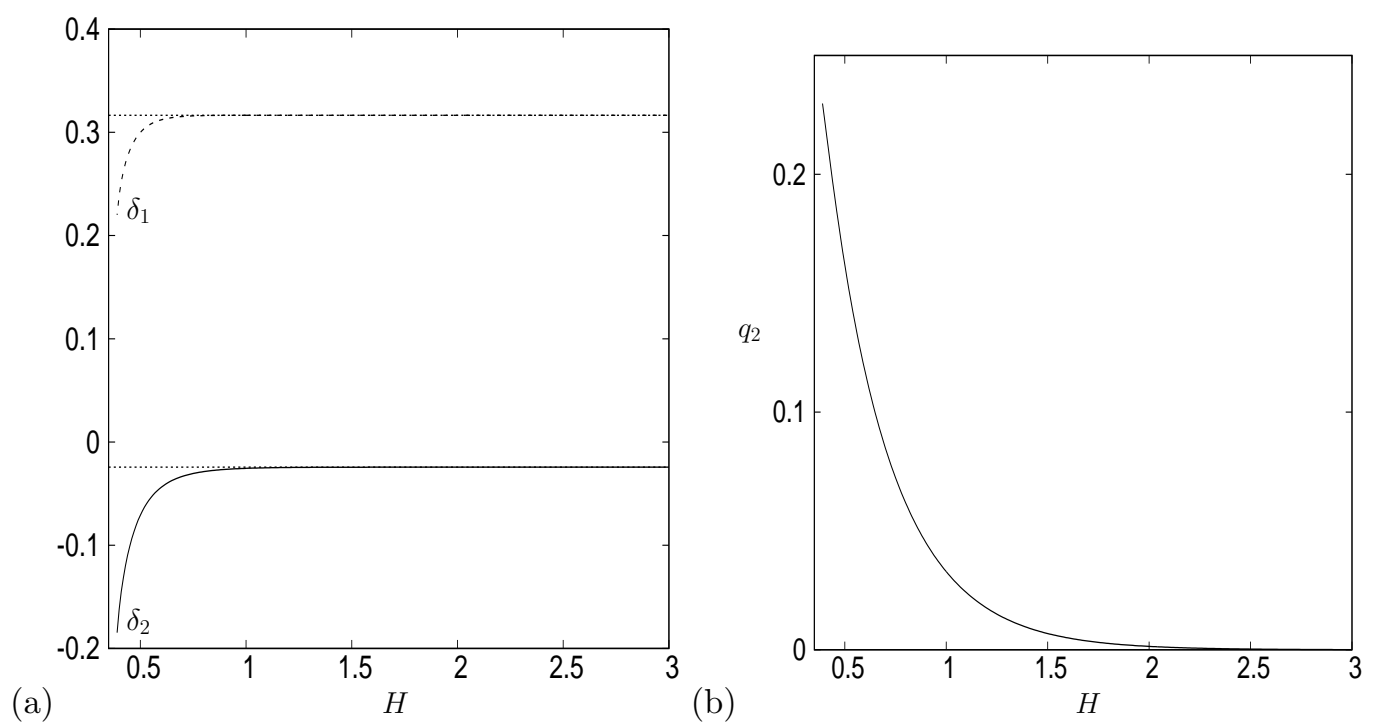

Fig. 14 Plot of (a) $\delta_{1}(H)$ and $\delta_{2}(H)$ and (b) $q_{2}(H)$ for the conformal mapping for the scenario $N=1$ baffle in finite depth, with $\epsilon=10^{-2}$. Here $q_{1}=0.166, \alpha=-0.5$ and $t=0$, and the horizontal dotted lines correspond to $\delta_{1}=0.3165$ and $\alpha=-0.0224$ from the infinite depth calculation.

does in the infinite depth map (2.5). This is not totally unexpected though, as both $c^{(2)}$ in finite depth and $\alpha$ in infinite depth get mapped to the origin in the $\eta$-plane. This however, then means that the parameter $\alpha$ in the finite depth mapping becomes arbitrary, except for the fact that it must lie inside $c^{(0)}$, on the real axis, and not on the inversion contour $\mathscr{C}$ of Cauchy's integral theorem. The resulting conformal mapping for $\alpha=-0.5$ and $H=0.5$ is plotted in Fig. 15. The results in Fig. 14 show that the infinite depth limit occurs for $H \gtrsim 1$.

In Fig. 16 we investigate the restriction on the arbitrary mapping parameter $q_{1}$ for the case depicted in Fig. 15 for $H=1$ and $H=0.5$ with $\alpha=-0.5$. For the similar result in infinite depth (see Fig. 8) we only found solutions for the mapping when $t=0$ and $\epsilon=10^{-4}$, for $q_{1} \in[0.1658,0.1817]$. In the finite depth case this restriction is relaxed and we can compute the mapping for $q_{1} \in(0,0.1881]$ for $H=1$ and $q_{1} \in(0,0.1860]$ for $H=0.5$. In both cases $\delta_{1}$ and $\delta_{2}$ move towards 1 as $q_{1} \rightarrow 0$ and for $q_{1}>0$ the solutions cease when $\delta_{1}$ becomes zero. Therefore this shows that the finite depth mapping (2.6) appears to be more robust than the infinite depth mapping, and it is more likely that a solution map can be found because the available range of $q_{1}$ values is larger. As part of the numerical process, as we have currently defined it, involves choosing a value of $q_{1}$ in advance, the finite depth mapping makes it more likely that an appropriate value is chosen. This coupled with the results in Fig. 14, show that the mapping (2.6) should also be used for infinite depth calculations, with $H$ chosen appropriately large, as in this limit the range of possible $q_{1}$ values is still larger than those found in Fig. 8.

One final check to test the potential benefit of using the finite depth mapping (2.6) over the infinite depth mapping (2.5) is to calculate the time-dependent solution for the cases 

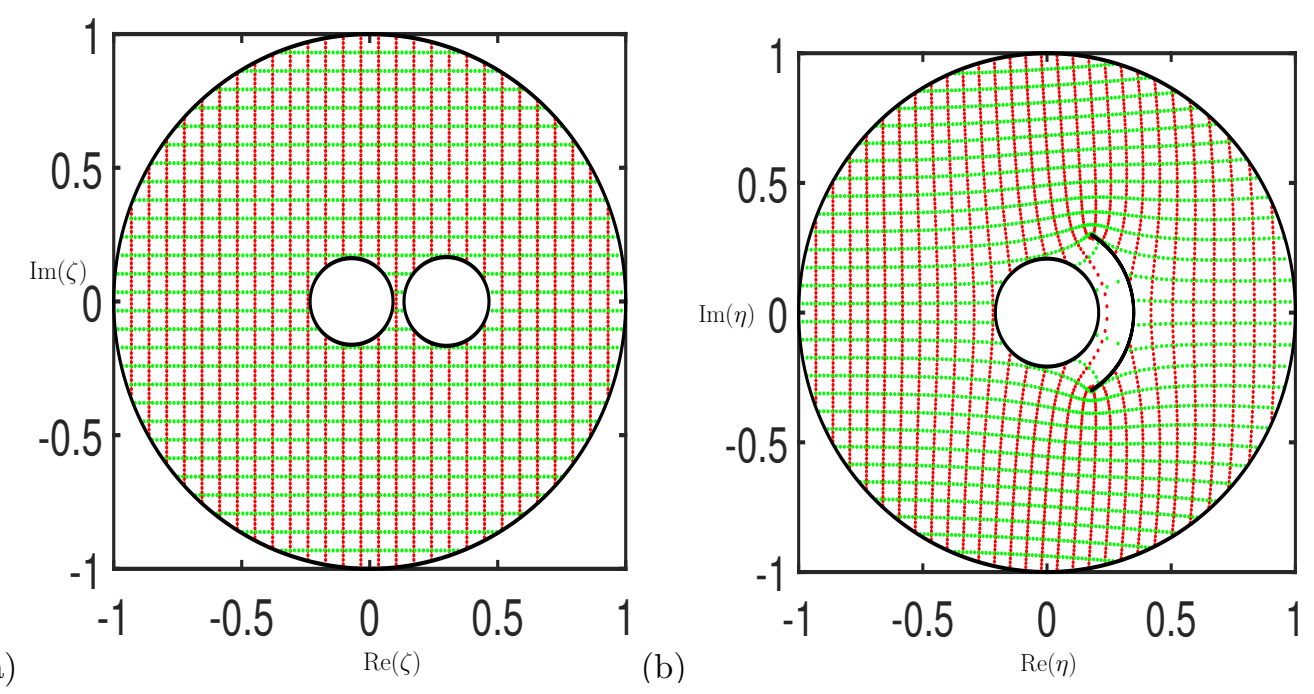

(a)

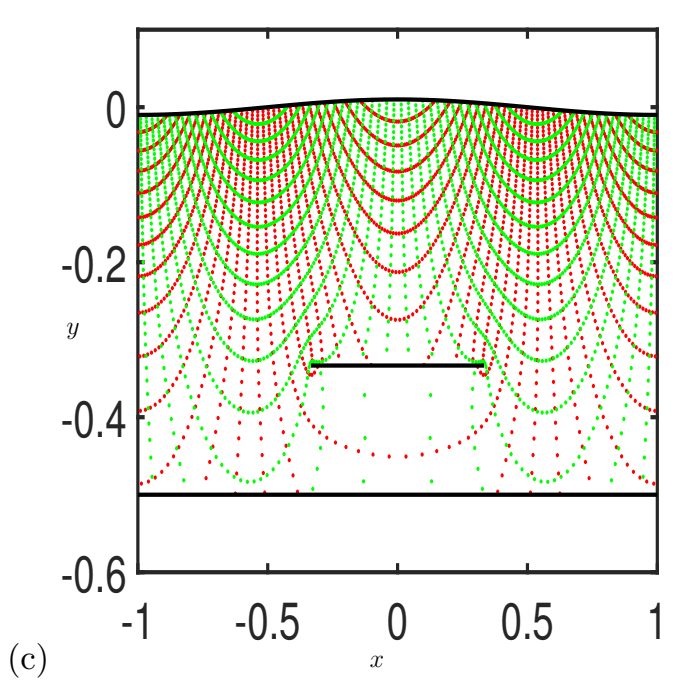

Fig. 15 Plot of (a) the $\zeta$-plane, (b) the $\eta$-plane and (c) the $z$-plane showing the conformal mapping for the scenario $N=1$ baffle in finite depth for $\epsilon=10^{-2}, H=0.5$ and $t=0$ in (4.1). Here we have set $q_{1}=0.166$ and $\alpha=-0.5$.

considered in Fig. 16. For the infinite depth scenario the solution when $q_{1}=0.166$ ceased at $t=1.634$ when $\alpha$ became zero (see case 1 in Fig. 11). This time, with $\delta_{2}$ behaving as $\alpha$ from the infinite depth mapping, we find that time-dependent results are possible for all $t \in[0, \pi]$.

To complete this study we plot the infinite-depth conformal maps from Fig. 12 in finite depth with $H=0.6$ in Fig. 18. The mappings are very similar to those in infinite depth, with some slight variations around the baffles. The final thing to note here is that the 

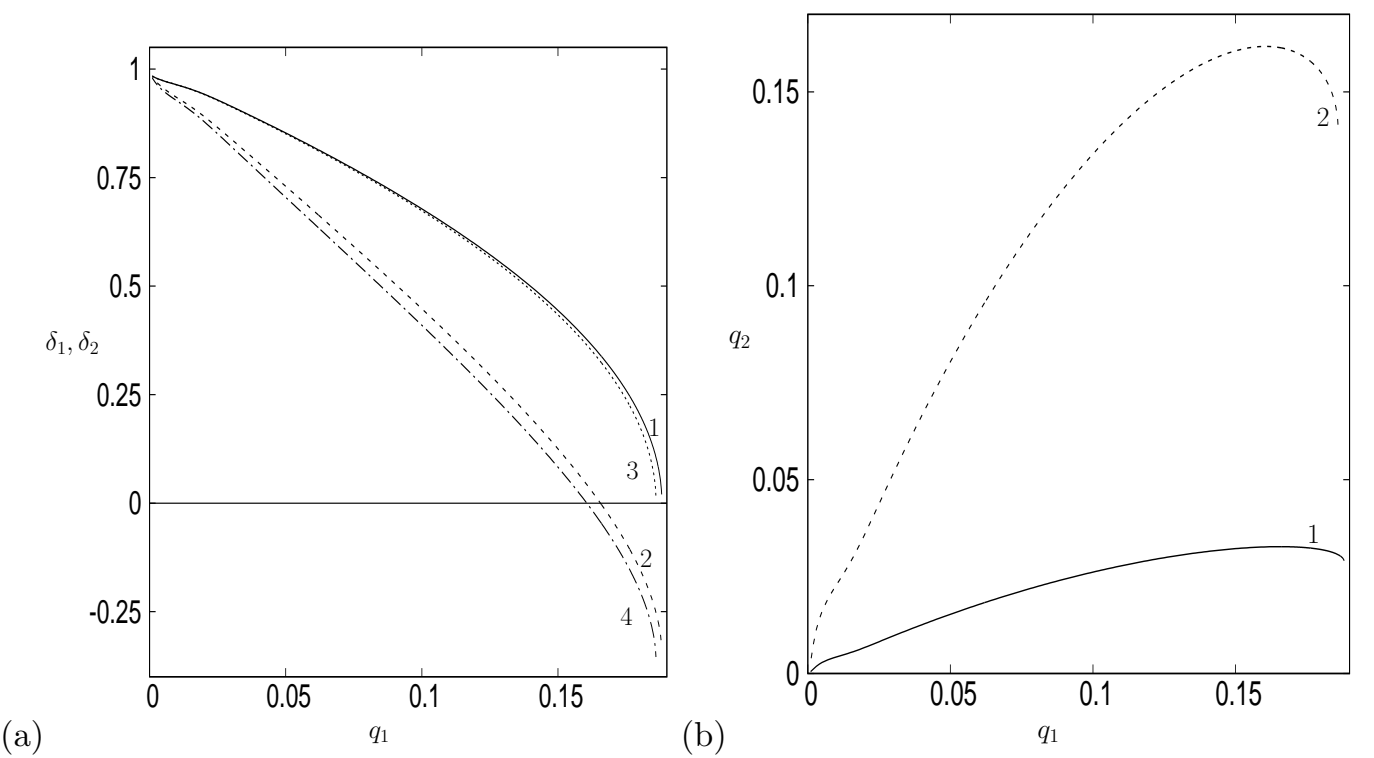

Fig. 16 Panel (a) $\delta_{1}\left(q_{1}\right)$ (results 1 and 3) and $\delta_{2}\left(q_{1}\right)$ (results 2 and 4 ) for $H=1$ (results 1 and 2) and $H=0.5$ (results 3 and 4). Panel (b) $q_{2}\left(q_{1}\right)$ for $H=1$ (result 1) and $H=0.5$ (result 2). Both panels are for the scenario of $N=1$ baffle in finite depth with $\alpha=-0.5, \epsilon=10^{-4}$ and $t=0$.

parameter $\alpha$ from (2.6) is arbitrary, but as stated above, must be chosen to lie inside $c^{(0)}$, on the real axis and not on $\mathscr{C}$ of (2.7). For the cases in panels (a) and (b) this is straight forward and $\alpha$ is chosen to lie significantly far from the origin along the negative real axis, but in panels (c) and (d) it must lie between $c^{(2)}$ and $c^{(3)}$ in the $\zeta$-plane. This sounds straight forward, but these large amplitude results are obtained using parameter continuation with $\epsilon$ increased from $10^{-3}$ to $10^{-2}$ (the code would not converge with $\epsilon=10^{-2}$ with zero initial guesses for $\widetilde{x}_{0}, \widetilde{x}_{1}, \widetilde{x}_{2}$ and $\widetilde{x}_{3}$ ), and so the circles $c^{(2)}$ and $c^{(3)}$ move during this process. If you are unlucky, then the overlap region of possible $\alpha$ values at $\epsilon=10^{-2}$ and $\epsilon=10^{-3}$ is the empty set, as is the case for Fig. 18(d). In this case we found it beneficial to also update $\alpha$ during the parameter continuation by placing $\alpha$ midway between at each iterative step $c^{(2)}$ and $c^{(3)}$ via

$$
\alpha=\frac{1}{2}\left(\delta_{2}+q_{2}+\delta_{3}-q_{3}\right) .
$$

\section{Conclusions and Discussion}

In this paper we performed a feasibility study investigating the suitability of using timedependent conformal mappings of multiply-connected domains to numerically simulate the evolution of the unknown free-surface in fully-dynamic free-surface flows. The particular flow investigated was that of an inviscid, irrotational, incompressible fluid sloshing in a rectangular vessel with rigid, impermeable baffles connected to the side-walls. We show that this flow can be mapped conformally to a $\zeta$-plane where the unknown free-surface is mapped to the unit circle and the surface of the $N$ baffles and the impermeable bottom 

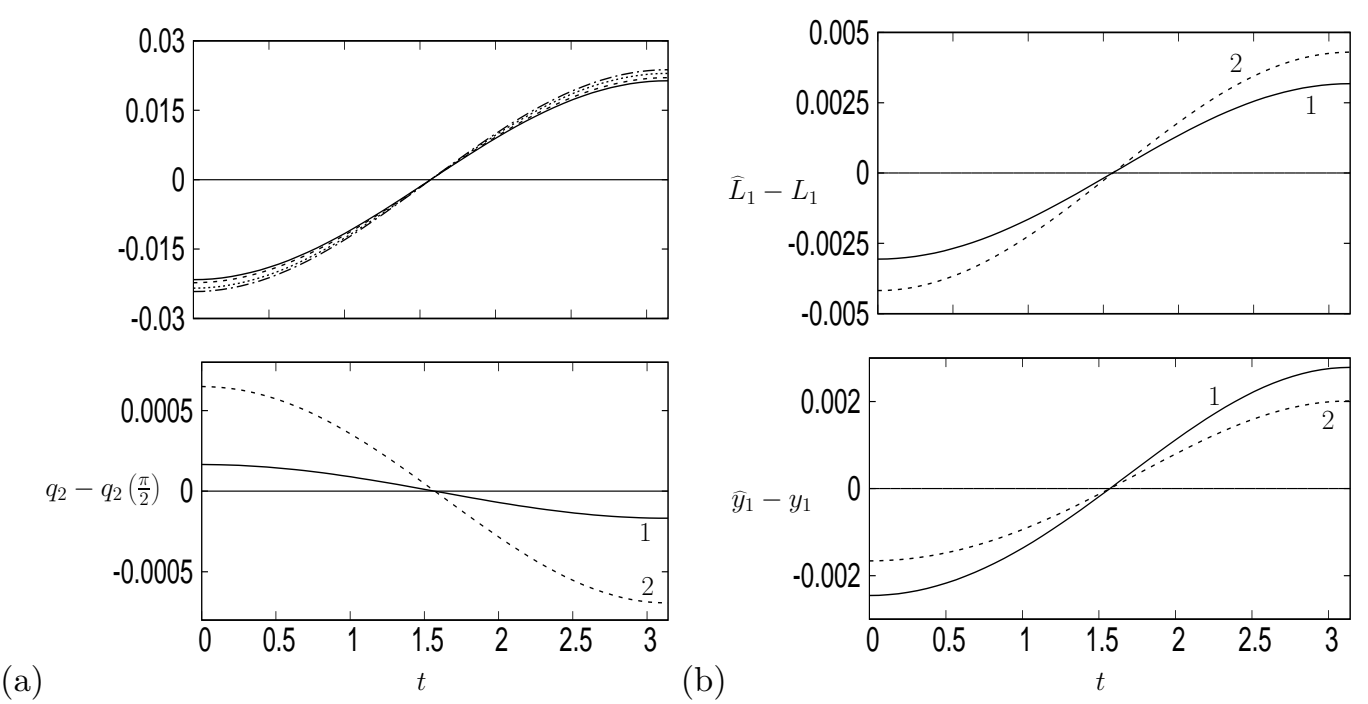

(a)

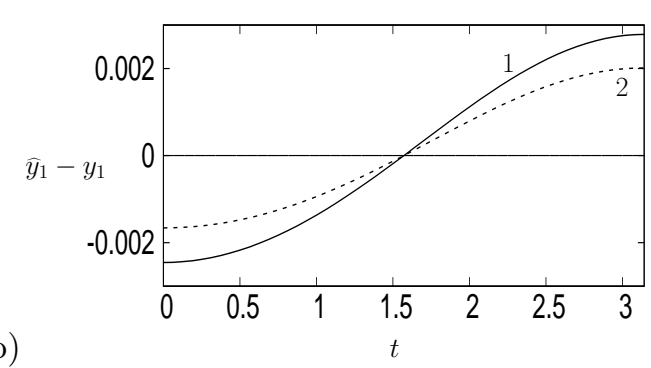

Fig. 17 For the scenario of $N=1$ baffle in finite depth with $\epsilon=10^{-2}$, plots of (a) $\delta_{1}(t)-\delta_{1}(\pi / 2)$, $\delta_{2}(t)-\delta_{2}(\pi / 2)$ (top panel) and $q_{2}(t)-q_{2}(\pi / 2)$ (bottom panel) and (b) $\widehat{L}_{1}(t)-L_{1}$ (top panel) and $\widehat{y}_{1}(t)-y_{1}$ (bottom panel). In the top panel of (a) results for $\delta_{1}(t)$ and $\delta_{2}(t)$ with $H=1$ and $H=0.5$ are almost indistinguishable, while in the other panels $H=1$ and $H=0.5$ correspond to results 1 and 2 respectively.

(in finite depth flows) are mapped to $N+1$ circles cut-out from this domain. Thus the domain is $(N+2)$-fold connected for a finite depth fluid $((N+1)$-fold connected in an infinite fluid). An algorithm is devised using Cauchy's integral theorem which relates the conjugate harmonic functions along the unknown free-surface. This algorithm could then be incorporated into time-dependent dynamic simulations to model the time-evolution of the free-surface. The algorithm makes use of the Schottky-Klein prime function in order to map from the $\zeta$-plane described above to one where the circles representing the baffles are mapped to circular arcs (23).

The results presented in the paper showed that for infinite depth fluids the conformal mapping contained one free parameter ( $q_{1}$ in our case) to be chosen by the user from a range of possible values. However for the finite depth conformal mapping there were two free parameters ( $q_{1}$ and $\alpha$ in our case) to be chosen which gave a wider tolerance to the user when determining these values for the initial iteration step. Results of the mappings are presented in both infinite and finite depth fluids, and the variation of the mapping parameters were examined as functions of the amplitude of the free-surface elevation and an imposed time variation, where this variation was chosen to simulate a wave sloshing from the left-hand wall of the vessel to the right-hand wall. The kinematic time-dependent results presented in infinite depth show that the sloshing wave can only complete its progress to the right-hand wall for a small range of $q_{1}$ values, where $q_{1}$ is fixed for the duration of the motion. This restriction can be lifted slightly if we allow $q_{1}$ to also vary in some prescribed way during the sloshing motion. However, in the dynamic time-dependent problem, when 
(a)

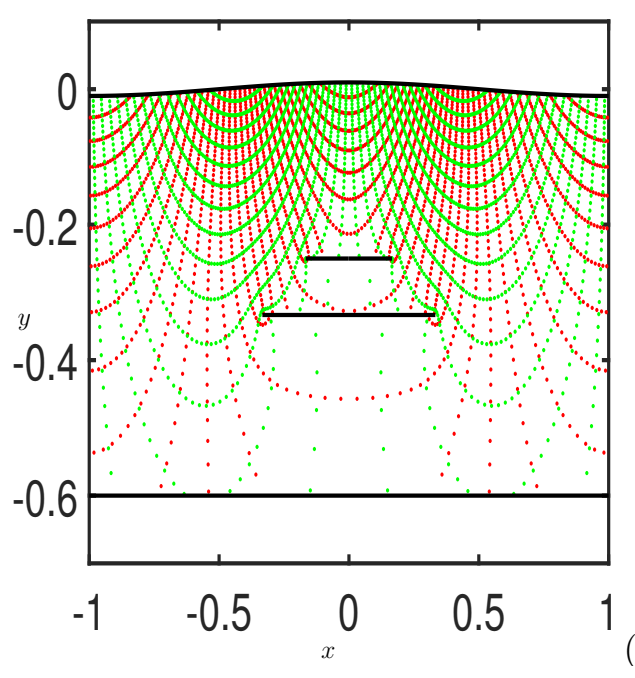

(b)
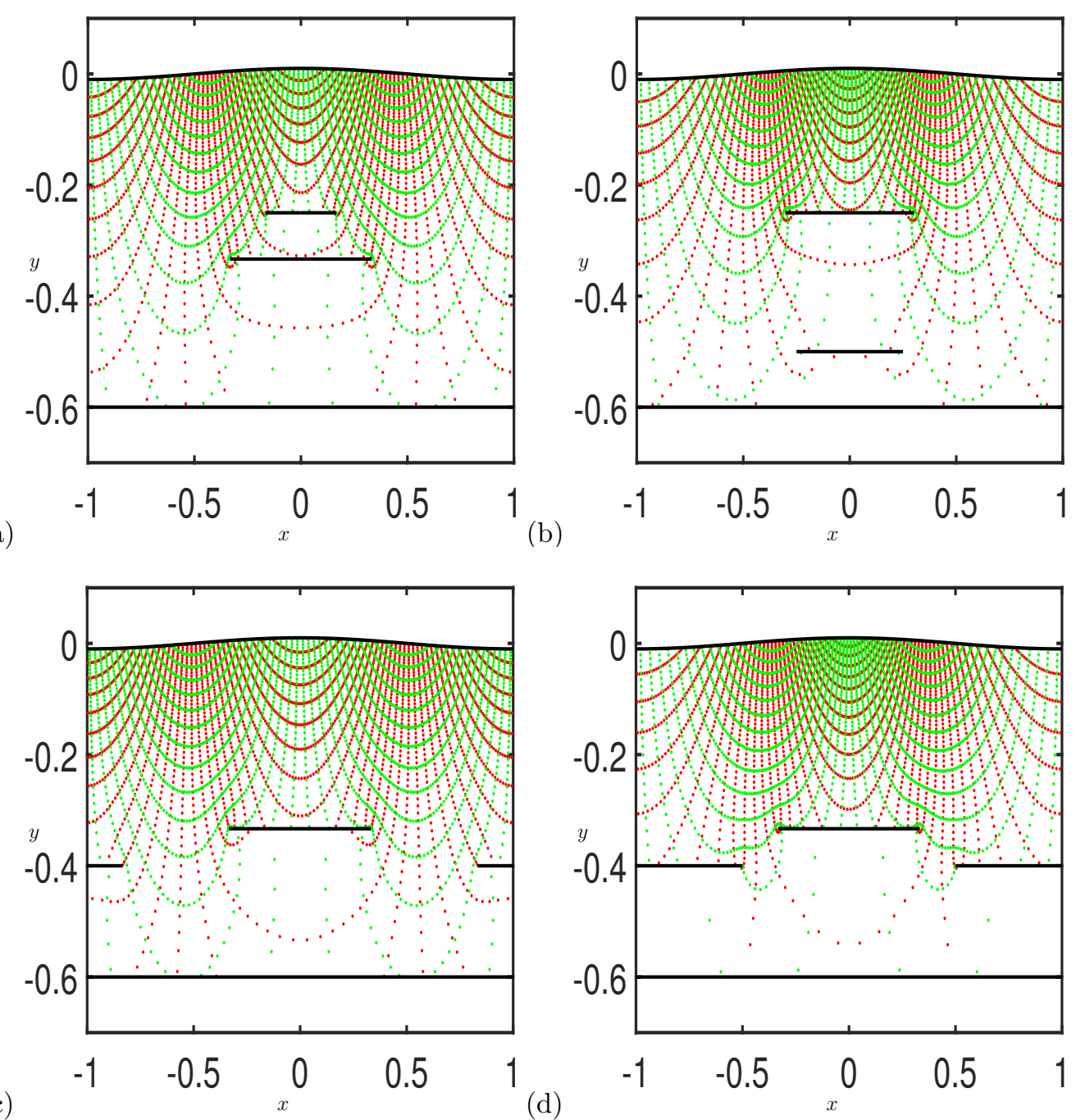

(d)

Fig. 18 Plot of the $z$-plane, showing the conformal mapping for the scenario $N=2$ baffles in finite depth with $H=0.6$ for $\epsilon=10^{-2}$ and $t=0$ in (4.1). The panels (a)-(d) correspond to the cases $5-8$ in table 2 respectively.

the free-surface is not known a priori, deciding how to vary $q_{1}$ is unclear and thus would be difficult to incorporate into a numerical algorithm which works in a wide range of situations. For the finite depth mapping this situation is more straightforward to deal with, because $q_{1}$ can be fixed throughout the duration of the motion, and $\alpha$ can be considered as timedependent with the property that it always lies midway between the centres of the circles in the $\zeta$-plane representing the bottom, $c^{(N+1)}$, and the lowest baffle on the right-hand wall. 
If there is no baffle on the right-hand wall then halfway between the centre of $c^{(N+1)}$ and the point -1 will suffice.

The reason for carrying out this feasibility study was to discover if conformal maps could be used to simulate the fully dynamic sloshing problem in a rectangular vessel with side-wall baffles, in order to calculate quantities such as the natural frequencies of the linear modes, as well as nonlinear free-surface profiles for larger amplitude motions. This conformal mapping approach has been utilized in the case without side-wall baffles in free sloshing (4), forced sloshing (33) and dynamic sloshing (34) problems. In the dynamic problems the parametric form of the free-surface from (2.1) is not known a priori and is governed by a pair of PDEs of the form

$$
\frac{\partial Y_{0}}{\partial t}=F_{1}\left(X_{0}, Y_{0}, \Phi, \Psi\right), \quad \frac{\partial \Phi}{\partial t}=F_{2}\left(X_{0}, Y_{0}, \Phi, \Psi\right),
$$

where $F_{1}$ and $F_{2}$ are functions (whose form for specific problems can be found in $(4 ; 33 ; 34)$ ). The complex potential $\phi+\mathrm{i} \psi$ is another analytic variable which can utilize the conformal mapping in this paper, to link the velocity potential, $\phi$, and the streamfunction, $\psi$, on the free-surface, given respectively by $\Phi(\mu)=\phi(\mu, 0)$ and $\Psi(\mu)=\psi(\mu, 0)$. Equations (5.1) are integrated forward in time using an explicit integration scheme from some initial condition, and at each time step, once the new form of $Y_{0}(\mu)$ and $\Phi(\mu)$ are calculated, the conformal mapping approach from this paper can be used to generate the conjugate harmonic functions $X_{0}(\mu)$ and $\Psi(\mu)$ at that time-step. The solution for $X_{0}(\mu)$ is calculated using the algorithm documented in this paper directly, while the solution of $\Psi(\mu)$ would require a slightly modified algorithm to solve the imaginary parts of the equations similar to $(2.9)$, i.e. determining the imaginary part of the conjugate harmonic function given the real part. The incorporation of this algorithm in dynamic simulations is currently under consideration in future work.

\section{Acknowledgements}

MRT would like to acknowledge Thomas J. Bridges for his insightful discussions during the production of this manuscript, and Rhodri B. Nelson and Darren G. Crowdy for their assistance with the MATLAB subroutines for evaluating the Schottky-Klein prime function.

\section{References}

[1] P. Henrici, Applied and Computational Complex Analysis, Volume III, John Wiley \& Sons (New York) (1993).

[2] N. Papamichael and N. Stylianopoulos, Numerical conformal mapping: Domain decomposition and the mapping of quadrilaterals, World Scientific (Singapore) (2010).

[3] I. E. Garrick, Potential flow about arbitrary biplane wing sections, NASA Technical Report 542 (1937) 1-29.

[4] M. R. Turner and T. J. Bridges, Time-dependent conformal mapping of doublyconnected regions, Adv. Comp. Math 42(4) (2016) 947-972.

[5] O. M. Faltinsen and A. N. Timokha, Sloshing, Cambridge University Press (Cambridge) (2009). 
[6] F. Dias and T. J. Bridges, The numerical computation of freely propagating timedependent irrotational water waves, Fluid Dyn. Res. 38(12) (2006) 803-830.

[7] J. G. B. Byatt-Smith, An integral equation for unsteady surface waves and a comment on the Boussinesq equation, J. Fluid Mech. 49 (1971) 625-633.

[8] A. K. Whitney, The numerical solution of unsteady free surface flows by conformal mapping, Proc. 2nd Int. Conf. Numerical Methods in Fluid Dynamics (ed. M. Holt). Lecture Notes in Physics, vol 8, Springer (Berlin Heidelberg) (1971) 458-462.

[9] L. V. Ovsjannikov, A plane problem on the unsteady motion of an incompressible fluid with a free boundary, Dinamika Splošn. Sredy Vyp. 8 (1971) 22-26.

[10] M. A. Grant, Standing Stokes waves of maximum height, J. Fluid Mech. 60 (1975) 593604.

[11] V. V. Voinov and O. V. Voinov, Numerical method of calculating nonstationary motions of an ideal incompressible liquid with free surfaces, Sov. Phys. Dokl. 20 (1975) 179-180.

[12] S. Tanveer, Singularities in water waves and Rayleigh-Taylor instability, Proc. Roy. Soc. Lond. A 435 (1991) 137-158.

[13] A. I. Dyachenko, E. A. Kuznetsov, M. D. Spector and V. E. Zakharov, Analytical description of the free surface dynamics of an ideal fluid (canonical formalism and conformal mapping), Phys. Lett. A 221 (1996) 73-79.

[14] D. Chalikov and D. Sheinin, Numerical modeling of surface waves based on principal equations of potential wave dynamics, NOAA Tech. Rep. 139 (1996) 1-64.

[15] D. Chalikov and D. Sheinin, Modeling extreme waves based on equations of potential flow with a free surface, J. Comp. Phys. 210 (2005) 247-273.

[16] D. Chalikov, Numerical simulation of the Benjamin-Feir instability and its consequences, Phys. Fluids 19 (2007) 016602.

[17] R. V. Shamin, Dynamics of an ideal liquid with a free surface in conformal variables, J. Math. Sci. 160(5) (2009) 537-678.

[18] P. A. Milewski, J. M. Vanden-Broeck and Z. Wang, Dynamics of steep two-dimensional gravity-capillary waves, J. Fluid Mech. 664 (2010) 466-477.

[19] A. I. Dyachenko, V. E. Zakharov and E. A. Kuznetsov, Nonlinear dynamics of the free surface of an ideal fluid, Plasma Phys. Rep. 22 (1999) 916-928.

[20] W. Choi and R. Camassa, Exact evolution equations for surface waves, J. Eng, Mech. 125 (1999) 756-760.

[21] Y. A. Li, J. M Hyman and W. Choi, A numerical study of the exact evolution equations for surface waves in water of finite depth, Stud. Appl. Math. 113 (2004) 303-324. 
[22] C. Viotti, D. Dutykh and F. Dias, The conformal-mapping method for surface gravity waves in the presence of variable bathymetry and mean current, Procedia IUTAM (2013) 1-13.

[23] D. G. Crowdy and J. Marshall, Conformal mappings between canonical multiply connected domains, Comput. Methods Funct. Theory 6(1) (2006) 59-76.

[24] D.G. Crowdy and J. S. Marshall, Computing the Schottky-Klein prime function on the Schottky double of planar domains, Comput. Methods and Funct. Theory 7(1) (2007) 293-308.

[25] T. K. DeLillo and E. H. Kropf, Slit maps and Schwarz-Christoffel maps for multiply connected domains, Electron. Trans. Numer. Anal 36 (2010) 195-223.

[26] D. G. Crowdy, A. S. Fokas and C. C. Green, Conformal mappings to multiply connected polycircular arc domains, Comput. Methods Func. Theory 11(2) (2012) 685-706.

[27] D. G. Crowdy, Conformal slit maps in applied mathematics, ANZIAM J. 53(3) (2012) 171-189.

[28] D. G. Crowdy, E. H. Kropf, C. C. Green and M. M. S. Nasser, The Schottky-Klein prime function: a theoretical and computational tool for applications, IMA J.Appl. Math. 81(3) (2016) 589-628.

[29] M. Schiffer, Recent advances in the theory of conformal mapping, appendix to: R. Courant, Dirichlet's Principle, Conformal Mapping and Minimal Surfaces, (1950).

[30] Z. Nehari, Conformal mapping, Courier Corporation (1975).

[31] J.-M. Vanden-Broeck, Gravity-capillary free-surface flows, Cambridge University Press (Cambridge) (2010).

[32] J. Wilkening and J. Yu, Overdetermined shooting methods for computing standing water waves with spectral accuracy, Comp. Science and Discovery 5 (2012) 014017.

[33] M. R. Turner, Liquid sloshing in a horizontally forced vessel with bottom topography, J. Fluid Struct. 64 (2016) 1-26.

[34] M. R. Turner, T. J. Bridges and H. Alemi Ardakani, The pendulum-slosh problem: Simulation using a time-dependent conformal mapping, J. Fluid Struct. 59 (2015) 202223.

\section{APPENDIX A}

Governing integral equations for $N=1$ baffle in an infinite depth fluid

For the scenario of $N=1$ baffle in a finite depth fluid, Cauchy's integral theorem becomes

$$
\begin{aligned}
\widetilde{x}(\zeta)+\mathrm{i} \widetilde{y}(\zeta)= & \frac{1}{2 \pi} \int_{-\pi}^{\pi} \frac{\widetilde{x}_{0}(\theta)+\mathrm{i} \widetilde{y}_{0}(\theta)}{e^{\mathrm{i} \theta}-\zeta} e^{\mathrm{i} \theta} \mathrm{d} \theta-\frac{q_{1}}{2 \pi} \int_{0}^{2 \pi} \frac{\widetilde{x}_{1}(\phi)+\mathrm{i} \widetilde{y}_{1}(\phi)}{\delta_{1}+q_{1} e^{\mathrm{i} \phi}-\zeta} e^{\mathrm{i} \phi} \mathrm{d} \phi \\
& -\frac{q_{2}}{2 \pi} \int_{-\pi}^{\pi} \frac{\widetilde{x}_{2}(\theta)+\mathrm{i} \widetilde{y}_{2}(\theta)}{\delta_{2}+q_{2} e^{\mathrm{i} \theta}-\zeta} e^{\mathrm{i} \theta} \mathrm{d} \theta .
\end{aligned}
$$


Evaluating this on the free-surface, the baffle and the bottom, and halving the domain by assuming $\widetilde{y}_{0}, \widetilde{y}_{1}$ and $\widetilde{y}_{1}$ are even function about $\theta=0$ and $\phi=\pi$, while $\widetilde{x}_{0}, \widetilde{x}_{1}$ and $\widetilde{x}_{2}$ are odd functions leads to the six integral equations

$$
\begin{aligned}
& \widetilde{x}_{0}(\sigma)=\frac{1}{2 \pi} \mathscr{P} \mathscr{V} \int_{-\pi}^{0}\left[\cot \left[\frac{1}{2}(\theta-\sigma)\right]-\cot \left[\frac{1}{2}(\theta+\sigma)\right]\right] \widetilde{y}_{0}(\theta) \mathrm{d} \theta \\
& -\frac{q_{1}}{\pi} \int_{0}^{\pi}\left[\operatorname{Re}\left(F_{10}(\sigma, \phi)-F_{10}(\sigma, 2 \pi-\phi)\right) \widetilde{x}_{1}-\operatorname{Im}\left(F_{10}(\sigma, \phi)+F_{10}(\sigma, 2 \pi-\phi)\right) \widetilde{y}_{1}\right] \mathrm{d} \phi \\
& -\frac{q_{2}}{\pi} \int_{-\pi}^{0}\left[\operatorname{Re}\left(F_{20}(\sigma, \theta)-F_{20}(\sigma,-\theta)\right) \widetilde{x}_{2}-\operatorname{Im}\left(F_{20}(\sigma, \theta)+F_{20}(\sigma,-\theta)\right) \widetilde{y}_{2}\right] \mathrm{d} \theta \\
& \widetilde{y}_{0}(\sigma)=\frac{1}{\pi} \int_{-\pi}^{0} y_{0}(\theta) \mathrm{d} \theta-\frac{1}{2 \pi} \mathscr{P} \mathscr{V} \int_{-\pi}^{0}\left[\cot \left[\frac{1}{2}(\theta-\sigma)\right]+\cot \left[\frac{1}{2}(\theta+\sigma)\right]\right] \widetilde{x}_{0}(\theta) \\
& -\frac{q_{1}}{\pi} \int_{0}^{\pi}\left[\operatorname{Im}\left(F_{10}(\sigma, \phi)-F_{10}(\sigma, 2 \pi-\phi)\right) \widetilde{x}_{1}+\operatorname{Re}\left(F_{10}(\sigma, \phi)+F_{10}(\sigma, 2 \pi-\phi)\right) \widetilde{y}_{1}\right] \mathrm{d} \phi \\
& -\frac{q_{2}}{\pi} \int_{-\pi}^{0}\left[\operatorname{Im}\left(F_{20}(\sigma, \theta)-F_{20}(\sigma,-\theta)\right) \widetilde{x}_{2}+\operatorname{Re}\left(F_{20}(\sigma, \theta)+F_{20}(\sigma,-\theta)\right) \widetilde{y}_{2}\right] \mathrm{d} \theta \\
& \widetilde{x}_{1}(\psi)=\frac{1}{\pi} \int_{-\pi}^{0}\left[\operatorname{Re}\left(F_{01}(\psi, \theta)-F_{01}(\psi,-\theta)\right) \widetilde{x}_{0}-\operatorname{Im}\left(F_{01}(\psi, \theta)+F_{01}(\psi,-\theta)\right) \widetilde{y}_{0}\right] \mathrm{d} \theta \\
& -\frac{1}{2 \pi} \mathscr{P} \mathscr{V} \int_{0}^{\pi}\left[\cot \left[\frac{1}{2}(\theta-\psi)\right]-\cot \left[\frac{1}{2}(\theta+\psi)\right]\right] \widetilde{y}_{1}(\theta) \mathrm{d} \phi \\
& -\frac{q_{2}}{\pi} \int_{-\pi}^{0}\left[\operatorname{Re}\left(F_{21}(\psi, \theta)-F_{21}(\psi,-\theta)\right) \widetilde{x}_{2}-\operatorname{Im}\left(F_{21}(\psi, \theta)+F_{21}(\psi,-\theta)\right) \widetilde{y}_{2}\right] \mathrm{d} \theta(\mathrm{A} .4) \\
& \widetilde{y}_{1}(\psi)=\frac{1}{\pi} \int_{-\pi}^{0}\left[\operatorname{Im}\left(F_{01}(\psi, \theta)-F_{01}(\psi,-\theta)\right) \widetilde{x}_{0}+\operatorname{Re}\left(F_{01}(\psi, \theta)+F_{01}(\psi,-\theta)\right) \widetilde{y}_{0}\right] \mathrm{d} \theta \\
& -\frac{1}{\pi} \int_{0}^{\pi} \widetilde{y}_{1}(\phi) \mathrm{d} \phi+\frac{1}{2 \pi} \mathscr{P} \mathscr{V} \int_{0}^{\pi}\left[\cot \left[\frac{1}{2}(\phi-\psi)\right]-\cot \left[\frac{1}{2}(\phi+\psi)\right]\right] \widetilde{x}_{1}(\phi) \mathrm{d} \phi \\
& -\frac{q_{2}}{\pi} \int_{-\pi}^{0}\left[\operatorname{Im}\left(F_{02}(\psi, \theta)-F_{02}(\psi,-\theta)\right) \widetilde{x}_{2}+\operatorname{Re}\left(F_{02}(\psi, \theta)+F_{02}(\psi,-\theta)\right) \widetilde{y}_{2}\right] \mathrm{d} \theta(\mathrm{A} .5) \\
& \widetilde{x}_{2}(\sigma)=\frac{1}{\pi} \int_{-\pi}^{0}\left[\operatorname{Re}\left(F_{02}(\sigma, \theta)-F_{02}(\sigma,-\theta)\right) \widetilde{x}_{0}-\operatorname{Im}\left(F_{02}(\sigma, \theta)+F_{02}(\sigma,-\theta)\right) \widetilde{y}_{0}\right] \mathrm{d} \theta \\
& -\frac{q_{1}}{\pi} \int_{0}^{\pi}\left[\operatorname{Re}\left(F_{12}(\sigma, \phi)-F_{12}(\sigma, 2 \pi-\phi)\right) \widetilde{x}_{2}-\operatorname{Im}\left(F_{12}(\sigma, \phi)+F_{12}(\sigma, 2 \pi-\phi)\right) \widetilde{y}_{2}\right] \mathrm{d} \phi \\
& -\frac{1}{2 \pi} \mathscr{P} \mathscr{V} \int_{-\pi}^{0}\left[\cot \left[\frac{1}{2}(\theta-\sigma)\right]-\cot \left[\frac{1}{2}(\theta+\sigma)\right]\right] \widetilde{y}_{1}(\theta) \mathrm{d} \theta \\
& \widetilde{y}_{2}(\sigma)=\frac{1}{\pi} \int_{-\pi}^{0}\left[\operatorname{Im}\left(F_{02}(\sigma, \theta)-F_{02}(\sigma,-\theta)\right) \widetilde{x}_{0}+\operatorname{Re}\left(F_{02}(\sigma, \theta)+F_{02}(\sigma,-\theta)\right) \widetilde{y}_{0}\right] \mathrm{d} \theta \\
& -\frac{q_{1}}{\pi} \int_{0}^{\pi}\left[\operatorname{Im}\left(F_{12}(\sigma, \phi)-F_{12}(\sigma, 2 \pi-\phi)\right) \widetilde{x}_{1}+\operatorname{Re}\left(F_{12}(\sigma, \phi)+F_{12}(\sigma, 2 \pi-\phi)\right) \widetilde{y}_{1}\right] \mathrm{d} \phi \\
& -\frac{1}{\pi} \int_{-\pi}^{0} \widetilde{y}_{2}(\theta) \mathrm{d} \theta+\frac{1}{2 \pi} \mathscr{P} \mathscr{V} \int_{0}^{\pi}\left[\cot \left[\frac{1}{2}(\theta-\sigma)\right]-\cot \left[\frac{1}{2}(\theta+\sigma)\right]\right] \widetilde{x}_{2}(\theta) \mathrm{d} \theta,
\end{aligned}
$$

where $F_{n m}(t, s)$ is given in (2.10). Discretizing the equations via (3.1) and (3.2) and evaluating at 
the mid-points (3.3) and (3.4) gives

$$
\begin{aligned}
& \frac{1}{2}\left(\widetilde{x}_{0 j}+\widetilde{x}_{0(j+1)}\right)-\frac{1}{2 \pi} \sum_{k=1}^{N}\left[\cot \left[\frac{1}{2}\left(\theta_{k}-\sigma_{j}^{m}\right)\right]-\cot \left[\frac{1}{2}\left(\theta_{k}+\sigma_{j}^{m}\right)\right]\right] \widetilde{y}_{0 k} \Delta \theta \\
& +\frac{q_{1}}{\pi} \sum_{k=1}^{2 M}\left[\operatorname{Re}\left(F_{10}\left(\sigma_{j}^{m}, \phi_{k}\right)-F_{10}\left(\sigma_{j}^{m}, 2 \pi-\phi_{k}\right)\right) \widetilde{x}_{1 k}-\operatorname{Im}\left(F_{10}\left(\sigma_{j}^{m}, \phi_{k}\right)+F_{10}\left(\sigma_{j}^{m}, 2 \pi-\phi_{k}\right)\right) \widetilde{y}_{1 k}\right] \Delta \phi \\
& \left.+\frac{q_{2}}{\pi} \sum_{k=1}^{N}\left[\operatorname{Re}\left(F_{20}\left(\sigma_{j}^{m}, \theta_{k}\right)-F_{20}\left(\sigma_{j}^{m},-\theta_{k}\right)\right) \widetilde{x}_{2 k}-\operatorname{Im}\left(F_{20}\left(\sigma_{j}^{m}, \theta_{k}\right)+F_{20}\left(\sigma_{j}^{m},-\theta_{k}\right)\right) \widetilde{y}_{2 k}\right] \Delta \theta=\alpha, \text { A.8 }\right) \\
& \frac{1}{2}\left(\widetilde{y}_{0 j}+\widetilde{y}_{0(j+1)}\right)-\frac{1}{\pi} \sum_{k=1}^{N} y_{0 k} \Delta \theta+\frac{1}{2 \pi} \sum_{k=1}^{N}\left[\cot \left[\frac{1}{2}\left(\theta_{k}-\sigma_{j}^{m}\right)\right]+\cot \left[\frac{1}{2}\left(\theta_{k}+\sigma_{j}^{m}\right)\right]\right] \widetilde{x}_{0 k} \\
& +\frac{q_{1}}{\pi} \sum_{k=1}^{2 M}\left[\operatorname{Im}\left(F_{10}\left(\sigma_{j}^{m}, \phi_{k}\right)-F_{10}\left(\sigma_{j}^{m}, 2 \pi-\phi_{k}\right)\right) \widetilde{x}_{1 k}+\operatorname{Re}\left(F_{10}\left(\sigma_{j}^{m}, \phi_{k}\right)+F_{10}\left(\sigma_{j}^{m}, 2 \pi-\phi_{k}\right)\right) \widetilde{y}_{1 k}\right] \Delta \phi \\
& \left.+\frac{q_{2}}{\pi} \sum_{k=1}^{N}\left[\operatorname{Im}\left(F_{20}\left(\sigma_{j}^{m}, \theta_{k}\right)-F_{20}\left(\sigma_{j}^{m},-\theta_{k}\right)\right) \widetilde{x}_{2 k}+\operatorname{Re}\left(F_{20}\left(\sigma_{j}^{m}, \theta_{k}\right)+F_{20}\left(\sigma_{j}^{m},-\theta_{k}\right)\right) \widetilde{y}_{2 k}\right] \Delta \theta=\alpha, \mathrm{A} .9\right) \\
& \frac{1}{2}\left(\widetilde{x}_{1 j}+\widetilde{x}_{1(j+1)}\right)-\frac{1}{\pi} \sum_{k=1}^{N}\left[\operatorname{Re}\left(F_{01}\left(\psi_{j}^{m}, \theta_{k}\right)-F_{01}\left(\psi_{j}^{m},-\theta_{k}\right)\right) \widetilde{x}_{0 k}-\operatorname{Im}\left(F_{01}\left(\psi_{j}^{m}, \theta_{k}\right)+F_{01}\left(\psi_{j}^{m},-\theta_{k}\right)\right) \widetilde{y}_{0 k}\right] \Delta \theta \\
& +\frac{1}{2 \pi} \sum_{k=1}^{2 M}\left[\cot \left[\frac{1}{2}\left(\theta_{k}-\psi_{j}^{m}\right)\right]-\cot \left[\frac{1}{2}\left(\theta_{k}+\psi_{j}^{m}\right)\right]\right] \widetilde{y}_{1 k} \Delta \phi \\
& +\frac{q_{2}}{\pi} \sum_{k=1}^{N}\left[\operatorname{Re}\left(F_{21}\left(\psi_{j}^{m}, \theta_{k}\right)-F_{21}\left(\psi_{j}^{m},-\theta_{k}\right)\right) \widetilde{x}_{2 k}-\operatorname{Im}\left(F_{21}\left(\psi_{j}^{m}, \theta_{k}\right)+F_{21}\left(\psi_{j}^{m},-\theta_{k}\right)\right) \widetilde{y}_{2 k}\right] \Delta \theta=0, \\
& \frac{1}{2}\left(\widetilde{y}_{1 j}+\widetilde{y}_{1(j+1)}\right)-\frac{1}{\pi} \sum_{k=1}^{N}\left[\operatorname{Im}\left(F_{01}\left(\psi_{j}^{m}, \theta_{k}\right)-F_{01}\left(\psi_{j}^{m},-\theta_{k}\right)\right) \widetilde{x}_{0 k}+\operatorname{Re}\left(F_{01}\left(\psi_{j}^{m}, \theta_{k}\right)+F_{01}\left(\psi_{j}^{m},-\theta_{k}\right)\right) \widetilde{y}_{0 k}\right] \Delta \theta \\
& +\frac{1}{\pi} \sum_{k=1}^{2 M} \widetilde{y}_{1 k} \Delta \phi-\frac{1}{2 \pi} \sum_{k=1}^{2 M}\left[\cot \left[\frac{1}{2}\left(\phi_{k}-\psi_{j}^{m}\right)\right]-\cot \left[\frac{1}{2}\left(\phi_{k}+\psi_{j}^{m}\right)\right]\right] \widetilde{x}_{1 k} \Delta \phi \\
& +\frac{q_{2}}{\pi} \sum_{k=1}^{N}\left[\operatorname{Im}\left(F_{02}\left(\psi_{j}^{m}, \theta_{k}\right)-F_{02}\left(\psi_{j}^{m},-\theta_{k}\right)\right) \widetilde{x}_{2 k}+\operatorname{Re}\left(F_{02}\left(\psi_{j}^{m}, \theta_{k}\right)+F_{02}\left(\psi_{j}^{m},-\theta_{k}\right)\right) \widetilde{y}_{2 k}\right] \Delta \theta=0, \\
& \frac{1}{2}\left(\widetilde{x}_{2 j}+\widetilde{x}_{2(j+1)}\right)-\frac{1}{\pi} \sum_{k=1}^{N}\left[\operatorname{Re}\left(F_{02}\left(\sigma_{j}^{m}, \theta_{k}\right)-F_{02}\left(\sigma_{j}^{m},-\theta_{k}\right)\right) \widetilde{x}_{0 k}-\operatorname{Im}\left(F_{02}\left(\sigma_{j}^{m}, \theta_{k}\right)+F_{02}\left(\sigma_{j}^{m},-\theta_{k}\right)\right) \widetilde{y}_{0 k}\right] \Delta \theta \\
& +\frac{q_{1}}{\pi} \sum_{k=1}^{2 M}\left[\operatorname{Re}\left(F_{12}\left(\sigma_{j}^{m}, \phi_{k}\right)-F_{12}\left(\sigma_{j}^{m}, 2 \pi-\phi_{k}\right)\right) \widetilde{x}_{2 k}-\operatorname{Im}\left(F_{12}\left(\sigma_{j}^{m}, \phi_{k}\right)+F_{12}\left(\sigma_{j}^{m}, 2 \pi-\phi_{k}\right)\right) \widetilde{y}_{2 k}\right] \Delta \phi \\
& +\frac{1}{2 \pi} \sum_{k=1}^{N}\left[\cot \left[\frac{1}{2}\left(\theta_{k}-\sigma_{j}^{m}\right)\right]-\cot \left[\frac{1}{2}\left(\theta_{k}+\sigma_{j}^{m}\right)\right]\right] \widetilde{y}_{1 k} \Delta \theta=0, \\
& \frac{1}{2}\left(\widetilde{y}_{2 j}+\widetilde{y}_{2(j+1)}\right)-\frac{1}{\pi} \sum_{k=1}^{N}\left[\operatorname{Im}\left(F_{02}\left(\sigma_{j}^{m}, \theta_{k}\right)-F_{02}\left(\sigma_{j}^{m},-\theta_{k}\right)\right) \widetilde{x}_{0 k}+\operatorname{Re}\left(F_{02}\left(\sigma_{j}^{m}, \theta_{k}\right)+F_{02}\left(\sigma_{j}^{m},-\theta_{k}\right)\right) \widetilde{y}_{0 k}\right] \Delta \theta \\
& +\frac{q_{1}}{\pi} \sum_{k=1}^{2 M}\left[\operatorname{Im}\left(F_{12}\left(\sigma_{j}^{m}, \phi_{k}\right)-F_{12}\left(\sigma_{j}^{m}, 2 \pi-\phi_{k}\right)\right) \widetilde{x}_{1 k}+\operatorname{Re}\left(F_{12}\left(\sigma_{j}^{m}, \phi_{k}\right)+F_{12}\left(\sigma_{j}^{m}, 2 \pi-\phi_{k}\right)\right) \widetilde{y}_{1 k}\right] \Delta \phi \\
& +\frac{1}{\pi} \sum_{k=1}^{N} \widetilde{y}_{2 k} \Delta \theta+\frac{1}{2 \pi} \sum_{k=1}^{N}\left[\cot \left[\frac{1}{2}\left(\theta_{k}-\sigma_{j}^{m}\right)\right]-\cot \left[\frac{1}{2}\left(\theta_{k}+\sigma_{j}^{m}\right)\right]\right] \widetilde{x}_{2 k} \Delta \theta=0,
\end{aligned}
$$



where $\Delta \theta=\pi / N$ and $\Delta \phi=\pi /(2 M)$. 